\title{
Diagnosis and treatment of cardiac amyloidosis: position statement of the German Cardiac Society (DGK)
}

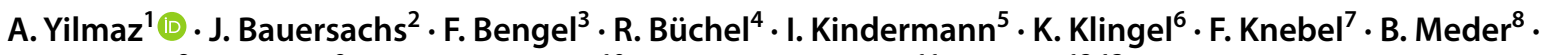

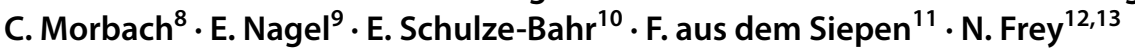

Received: 30 October 2020 / Accepted: 21 December 2020 / Published online: 18 January 2021

(c) The Author(s) 2021

\begin{abstract}
Systemic forms of amyloidosis affecting the heart are mostly light-chain (AL) and transthyretin (ATTR) amyloidoses. The latter is caused by deposition of misfolded transthyretin, either in wild-type (ATTRwt) or mutant (ATTRv) conformation. For diagnostics, specific serum biomarkers and modern non-invasive imaging techniques, such as cardiovascular magnetic resonance imaging (CMR) and scintigraphic methods, are available today. These imaging techniques do not only complement conventional echocardiography, but also allow for accurate assessment of the extent of cardiac involvement, in addition to diagnosing cardiac amyloidosis. Endomyocardial biopsy still plays a major role in the histopathological diagnosis and subtyping of cardiac amyloidosis. The main objective of the diagnostic algorithm outlined in this position statement is to detect cardiac amyloidosis as reliably and early as possible, to accurately determine its extent, and to reliably identify the underlying subtype of amyloidosis, thereby enabling subsequent targeted treatment.
\end{abstract}

Keywords Amyloidosis $\cdot$ Myocardium $\cdot$ Magnetic resonance imaging $\cdot$ Scintigraphy $\cdot$ Endomyocardial biopsy

Supplementary Information The online version contains supplementary material available at https://doi.org/10.1007/s0039 2-020-01799-3.

Major parts of this article were previously published in German language in "Der Kardiologe" (Yilmaz, A., Bauersachs, J., Kindermann, I. et al. Kardiologe (2019) 13:264. https://doi. org/10.1007/s12181-019-00344-5). The present article is an updated version of the aforementioned publication.

A. Yilmaz

ali.yilmaz@ukmuenster.de

1 Sektion für Herzbildgebung, Klinik für Kardiologie, Universitätsklinikum Münster, Von-Esmarch-Str. 48, 48149 Münster, Germany

2 Klinik für Kardiologie und Angiologie, Medizinische Hochschule Hannover, Hannover, Germany

3 Klinik für Nuklearmedizin, Medizinische Hochschule Hannover, Hannover, Germany

4 Klinik für Nuklearmedizin, Universitätsspital Zürich, Zurich, Switzerland

5 Klinik für Innere Medizin III (Kardiologie, Angiologie und Internistische Intensivmedizin), Universitätsklinikum des Saarlandes und Medizinische Fakultät der Universität des Saarlandes, Homburg, Germany
6 Institut für Pathologie und Neuropathologie, Universität Tübingen, Tübingen, Germany

7 Medizinische Klinik m.S. Kardiologie und Angiologie, Charite Universitätsmedizin Berlin Campus Mitte, Berlin, Germany

8 Klinik für Innere Medizin III, Universitätsklinikum Heidelberg, Heidelberg, Germany

9 Interdisziplinäres Amyloidosezentrum Nordbayern, Deutsches Zentrum für Herzinsuffizienz, Medizinische Klinik I der Universität Würzburg, Würzburg, Germany

10 Institut für Experimentelle und translationale kardiovaskuläre Bildgebung, Universitätsklinikum Frankfurt, Frankfurt, Germany

11 Institut für Genetik von Herzerkrankungen (IfGH), Universitätsklinikum Münster, Münster, Germany

12 Klinik für Innere Medizin III, Schwerpunkt Kardiologie und Angiologie, Universitätsklinikum Schleswig-Holstein, Kiel, Germany

13 Kommission für Klinische Kardiovaskuläre Medizin, Deutsche Gesellschaft für Kardiologie, Düsseldorf, Germany 


\section{Preamble}

This position statement is the first expert opinion on the diagnosis and treatment of cardiac amyloidosis written by members of the German Cardiac Society (Deutsche Gesellschaft für Kardiologie-Herz- und Kreislaufforschung, DGK).

The information contained in this position statement reflects a systematic review of the current state of knowledge on this issue. In addition, based on the evaluation of current clinical studies and the expertise of the authors involved, clinical recommendations are formulated to sensitize physicians to this disease and, if necessary, to provide support for everyday clinical practice.

Until a few years ago, amyloidosis was still considered a rare multi-organ disease with primarily neurological or hematological manifestation. Consequently, patients were treated primarily by neurologists and hematologists. Due to the heterogeneity of the symptoms, cardiac involvement of this disease was often overlooked or only documented at a late stage of disease-with unfavourable consequences for the prognosis of the affected patients. Meanwhile, it has become evident that "cardiac amyloidosis" is a frequent and, in some patients, the only manifestation, so that early cardiac diagnosis and subsequent cardiac-directed therapy are becoming increasingly important.

In this context, an interdisciplinary cooperation between specialists in neurology, hematology, gastroenterology, pathology, genetics, and cardiology (and other specialist groups) is an indispensable prerequisite for successful care of patients, and the establishment of specialized and interdisciplinary amyloidosis centres-with central involvement of cardiology—is highly useful.

While promising therapies for the treatment of the neurological manifestation were introduced some years ago, specific therapies for the treatment of cardiac amyloidosis have so far hardly been available. However, the targeted treatment of cardiac amyloidosis is increasingly the focus of clinical trials, and in addition to an extension of the label for already available drugs, the approval of new drugs is expected in the near future.

\section{Methodology of this position statement}

The PubMed database was searched for literature published until 08/2020. Scientific publications in German or English language were searched for the keywords "amyloidosis" and "cardiac amyloidosis", and evaluated for the assessment of the position statement.

It must be noted that prospective and randomized studies on cardiac amyloidosis with a reliable case numberwithout pharmaceutical sponsorship—are hardly available.
It must also be emphasized that some retrospective (partly multi-centre) studies with quite a large number of cases, which have been published in renowned journals and are often cited, have relevant methodological weaknesses. In this respect, a critical assessment of the available data and careful recommendations for the diagnosis and therapy of amyloidosis were necessary when preparing this position statement.

\section{Background}

\section{Definition and classification of amyloidoses}

"Amyloidosis" describes a disease entity caused by the pathologic deposition of misfolded proteins. In these proteins, instead of an alpha-helix conformation, there is a protein misfolding into a beta-leaf structure and the formation of insoluble fibrils, which are deposited in the interstitium of various organs. Destruction of the physiological tissue structure and direct toxicity of the amyloidogenic substrates lead to consecutive organ dysfunction [1].

Amyloidosis affects both sexes and individuals of any age. As a multi-organ disease presenting with a variety of rather unspecific symptoms of different severity, the diagnosis of amyloidosis is often delayed, and the number of undiagnosed cases is probably high.

So far, more than 30 different proteins have been described to cause, when misfolded, amyloidosis. There are localized forms (such as "alpha-atrial natriuretic factor" [AANF]-amyloidosis with deposition of atrial natriuretic peptide in the atria) and systemic forms such as AA amyloidosis in patients with chronic inflammation and deposition of serum amyloid A (SAA). The vast majority of systemic amyloidoses in industrialized countries are attributable to 3 subtypes: the most common form, which accounts for $\sim 70 \%$ of all amyloidoses, is light-chain (AL) amyloidosis, which occurs due to the deposition of misfolded immunoglobulin light chains mainly in the context of a monoclonal gammopathy or multiple myeloma. Less frequent are the two forms of the so-called ATTR amyloidosis caused by deposition of misfolded transthyretin (either as wild-type [ATTRwt] form or mutated/variant [ATTRv]) [2].

\section{Amyloidosis with cardiac involvement}

In principle, amyloid can be deposited in any organ and in any type of tissue. However, the different forms of amyloid show different organ tropism typical for the respective disease. Systemic forms of amyloidosis affecting the heart, are mainly AL, ATTRwt, and some forms of 
ATTRv amyloidosis. In the following, we will primarily focus on the diagnosis and therapy of cardiac relevant forms of amyloidosis.

Cardiac amyloidosis is typically associated with pronounced left-ventricular (LV) wall thickening ( $\geq 15 \mathrm{~mm}$ end diastolic), which can, however, be less pronounced in early stages $(<15 \mathrm{~mm})$. The term "hypertrophy" for this form of LV wall thickening is not quite correct, since "hypertrophy" is primarily a histological diagnosis and refers to an increased size of cardiomyocytes, whereas cardiac amyloidosis is characterized by wall thickening due to interstitial amyloid deposits. Nevertheless, the term "hypertrophy" has also become established in the literature for cardiac amyloidosis.

\section{Pathophysiology of AL amyloidosis}

The underlying cause of AL amyloidosis is usually a small clonal B cell or plasma cell population, whereas only about $10 \%$ of patients have overt multiple myeloma or, in rare cases, a secretory active B-cell lymphoma. In approximately $70 \%$ of AL amyloidosis cases, lambda light-chain expression can be found [3]. The cardiac dysfunction observed in AL amyloidosis is attributed to the interstitial deposition of the immunoglobulin light chains, but also to their direct toxicity. Amyloidogenic free light chains can induce in myocytes a lysosomal dysfunction, oxidative stress, apoptosis, and dysregulation of MAP kinase signaling transduction pathways and autophagy. These findings suggest that direct intracellular cytotoxic effects of immunoglobulin light chains are at least partially responsible for the rapid progression of the disease and poor prognosis [4].

\section{Pathophysiology of ATTRv and ATTRwt amyloidosis}

Transthyretin (TTR) is a transport protein for retinol and thyroid hormone synthesized by the liver, which circulates as a stable tetramer. Transthyretin shows a tendency to form amyloid fibrils when the tetramers dissociate into TTR monomers. The holoretinol-binding protein (RBP) binds and stabilizes tetrameric TTR, suggesting that low concentrations of RBP may be a risk factor for ATTR cardiomyopathy. The cleavage of the TTR tetramers into amyloidogenic monomers susceptible to aggregation is therefore regarded as a speed-determining step in amyloid formation [5]. The destabilization of tetramers and the accumulation of TTR monomers are either the consequence of gene mutations in the transthyretin (TTR) gene (ATTRv) or, if absent, are associated with age-related processes that are only partially understood (ATTRwt) [6]. It is unclear which factors explain the higher prevalence in men, the preferred accumulation in certain tissues and the later manifestation of ATTRwt, although the protein is present from birth.

To date, more than 100 mutations have been described in the TTR gene, although most ATTRv amyloidoses are caused by p.Val142Ile (also known as Val122Ile) and p.Val50Met (also known as Val30Met) mutations. These pathogenic amino acid substitutions induce conformational changes that destabilize the native tetramer structure and increase the likelihood of their dissociation in amyloidogenic TTR monomers. In this context, it is important to mention that, in addition to cardiotoxic TTR amyloid fibrils, non-fibrillary amyloidogenic TTR molecules are likely to have a cardiotoxic potential [7].

\section{Pathophysiology of AA amyloidosis}

AA amyloidosis is caused by the deposition of AA fibrils, which are formed from a fragment of the serum amyloid A (SAA) protein in the context of long-lasting inflammatory states. In contrast to ATTR amyloidosis, no mutations are known to promote the formation of fibrils. Nevertheless, there seems to be a genetic predisposition to develop AA amyloidosis. While AA amyloidosis mainly manifests in the gastrointestinal system and kidneys, heart involvement is rather rare. Important causes of AA amyloidosis are uncontrolled chronic inflammatory rheumatic diseases (e.g., rheumatoid arthritis), chronic inflammatory bowel diseases (ulcerative colitis, Crohn's disease), chronic infections (e.g., tuberculosis), and hereditary autoinflammatory diseases (e.g., familial Mediterranean fever).

In the liver, affected patients experience an increased production of the SAA protein, C-reactive protein (CRP), and other proteins of the acute phase reaction due to a chronic inflammatory condition under the influence of interleukin(IL)-1, IL-6, and tumor necrosis factor (TNF)- $\alpha$. SAA is coupled to lipoproteins $\left(\mathrm{HDL}_{\mathrm{SAA}}\right)$ and is taken up into the cell by HDL receptors. As a result of limited proteolysis of the SAA, the AA fragment is formed, which leads to the formation of a matrix in which the characteristic insoluble fibrils can form as a result of a change in conformation and interaction with the amyloid-enhancing factor (AEF), serum amyloid P (SAP), glycosaminoglycans, and other membrane proteins [8].

\section{Epidemiology of cardiac amyloidoses}

The exact prevalence and incidence of cardiac amyloidosis is unknown and varies greatly from region to region and between different ethnicities. In USA, a significant increase in the prevalence rate of cardiac amyloidosis among Medicare beneficiaries ( $8-17$ per 100,000 person-years) and incidence rate (18-55 per 100,000 person-years) was observed 
from 2000 to 2012 [9]. Moreover, approximately 2200 new cases of $\mathrm{AL}$ are diagnosed every year. The prevalence of $\mathrm{AL}$ amyloidosis has increased significantly (2.6-fold) between 2007 and 2015, from 15.5 cases per million in 2007 to 40.5 in 2015 [10]. Detailed information for Germany is not yet available. AL amyloidosis occurs in about $10 \%$ of patients suffering from multiple myeloma. Between 50 and $70 \%$ of patients with $\mathrm{AL}$ amyloidosis show cardiac involvement [6], which is decisive for morbidity and mortality, since in patients with untreated, advanced cardiac AL amyloidosis and heart failure, the mean survival time is only about 6 months [11]. In AL amyloidosis, modern treatment strategies have made it possible to extend the median overall survival to $>5$ years-at least in patients with less advanced stages [12].

ATTRwt amyloidosis predominantly affects the heart and shows a striking male dominance. The prevalence increases significantly with age: up to $25 \%$ of patients over 80 years of age show amyloid deposits in histopathological examinations [13]. Recent studies suggest that ATTRwt may be a causal factor in up to $10 \%$ of elderly patients with symptoms of heart failure [14]. In an autopsy study, 109 patients with heart failure and preserved ejection fraction (HFpEF) were examined, in which $19 \%$ of the patients showed amyloid deposits in the left ventricle, indicating that ATTRwt might play an etiologically important role also in HFpEF [15].

In contrast to ATTRwt, ATTRv amyloidosis is clinically quite variable in its prevalence and organ involvement depending on the specific TTR gene mutation: peripheral polyneuropathies are particularly common in addition to cardiac involvement. One of the most common mutations is the p.Val142Ile mutation, which occurs in 3-4\% of the African population, and the p.Val50Met mutation, which is prevalent in northern Portugal and Sweden. So far, no data exist on the TTR mutation frequency in German amyloidosis patients with cardiac involvement. The experience of most of the authors of this position statement suggests that the proportion of ATTRv amyloidoses among all ATTR amyloidoses in Germany is significantly lower than $10 \%$ and thus may differ from other European countries.

Detailed information on the frequency of AA amyloidosis is difficult to obtain. In fact, AA amyloidosis is possibly the most common form of amyloidosis worldwide, as it is associated with a variety of chronic inflammatory diseases. Registries report a prevalence of AA amyloidosis in about $10 \%$ of all patients with rheumatoid arthritis (RA). In a Finnish autopsy study of deceased RA patients, the frequency was reported to be up to $30 \%$ of all RA patients [16]. However, as anti-inflammatory therapeutic options improve, the prevalence of AA amyloidosis is likely to decrease.

\section{Clinical manifestation}

\section{Clinical and cardiac manifestations of AL amyloidosis}

When considering the total population, AL amyloidosis mainly affects the elderly, but may also affect younger patients in rare cases: the mean age at diagnosis is about 60 years. The clinical symptoms depend on the affected organs and the extent of organ involvement.

Non-specific symptoms such as weight loss, fatigue, dyspnoea on exertion, and oedema occur frequently [1]. Macroglossia with indentations in the lateral parts of the tongue, which is pathognomonic for AL amyloidosis and can lead to swallowing difficulties, and even life-threatening respiratory problems as well as a periorbital purpura (which typically occurs after minor injuries or physical exertion) are only detectable in a few patients with AL amyloidosis [17]. The most frequently affected organs are the heart (71-90\%), the kidneys (58-75\%), the gastrointestinal tract (22\%), the nervous system (23\%), and the liver (16-30\%) [1, 18]. The lungs and spleen can also be affected by amyloid deposits. Involvement of the soft tissue is less frequent (in 10-15\% of patients).

Cardiac involvement is characterized by symptoms of heart failure (peripheral oedema, dyspnoea, and pleural effusions) due to an existing diastolic and, in the further course, additionally occurring systolic dysfunction with a cardiac phenotype mostly resembling hypertrophic or restrictive cardiomyopathy. Arrhythmias can cause dizziness, syncope, or sudden cardiac death. Amyloid protein infiltration of the atria contributes to the high prevalence of atrial fibrillation and the increased risk of atrial thrombus formation in this disease [19]. In addition, amyloid can be deposited within and/or around the small arterioles of the heart, which can lead to clinical symptoms of angina pectoris or in some cases to myocardial infarction [20].

In patients with microvascular amyloid infiltration, a decrease in coronary flow reserve was detected by positron emission tomography (PET) [21].

Renal involvement may be demasked by proteinuria (with predominant albuminuria) and nephrotic syndrome with peripheral oedema, anasarca, foaming urine, or uremic signs. If the gastrointestinal tract is involved, symptoms such as cachexia, constipation, and diarrhoea (also alternating), heartburn, nausea, and vomiting may occur. Furthermore, there may be gastrointestinal bleeding. The so-called "shoulder pad sign" is characterized by periarticular amyloid deposits, which look like shoulder pads [1]. Progressive bilateral and symmetrical polyarthropathies of the fingers, wrists, and knees can also be caused by AL amyloidosis [18]. Furthermore, lymph-node enlargement may be detectable. 
If the nervous system is affected, mixed sensomotoric peripheral neuropathies (20\%) with numbness, paraesthesia, and pain up to paresis as well as autonomic neuropathies $(15 \%)$ with consecutive orthostatic hypotension will occur. Carpal tunnel syndrome is present in about $25 \%$ of patients and can occur years before diagnosis.

Coagulopathies such as a bleeding diathesis (e.g., due to an acquired factor $\mathrm{X}$ deficiency) or hypercoagulability can also be detected in patients with AL amyloidosis. Atrial thrombi often appear before the first overt manifestation of atrial fibrillation. In the case of an increased bleeding tendency without a detectable coagulation disorder, vascular involvement should be considered. The symptoms and clinical manifestations described so far may also appear only over the course of the disease [1].

\section{Disease progression and prognosis of $A L$ amyloidosis}

Cardiac amyloidosis is often overlooked and, due to the heterogeneity of the symptoms, the diagnosis is often made late, which in turn has unfavourable consequences for the prognosis of the patients. In a study of almost 500 patients with AL amyloidosis, $37 \%$ of the patients were diagnosed after $\geq 1$ year after the onset of initial symptoms, and a third of the patients visited 5 doctors before the diagnosis could be confirmed [22]. Further factors influencing the prognosis are the number of affected organs and the extent of organ involvement as well as the response to a specific therapy.

The differentiation of local from systemic AL amyloidosis is essential for prognostic assessment. Local amyloidosis, e.g., with isolated involvement of the skin and nails, the lungs, the eye, and the urogenital tract, has a good prognosis. The conversion of a local to a systemic amyloidosis is rare [1]. Cardiac involvement typically is the limiting factor in the mortality of AL amyloidosis and is the mortality reason in about $75 \%$ of patients.

The combination of the 3 biomarkers NT-proBNP, troponin $\mathrm{T}$, and circulating free light chains allows a risk stratification according to the "Mayo Clinic Staging System" ([23]; Table 1). In patients with atrial fibrillation or renal impairment, the modified Mayo Clinic Staging System (differentiating a stage IIIB if NT-proBNP $>8500 \mathrm{ng} / \mathrm{ml}$ ) performed best [24]. The NT-proBNP concentration also serves as a progression parameter for assessing the response to therapy or the progression of the disease. However, it must be taken into account that NT-proBNP concentration is dependent on kidney function, among others, and can be subject to fluctuations regardless of the cardiac condition if renal failure is present at the same time.

The prognosis of AL amyloidosis strongly depends on the disease stage and progression, respectively. In patients with

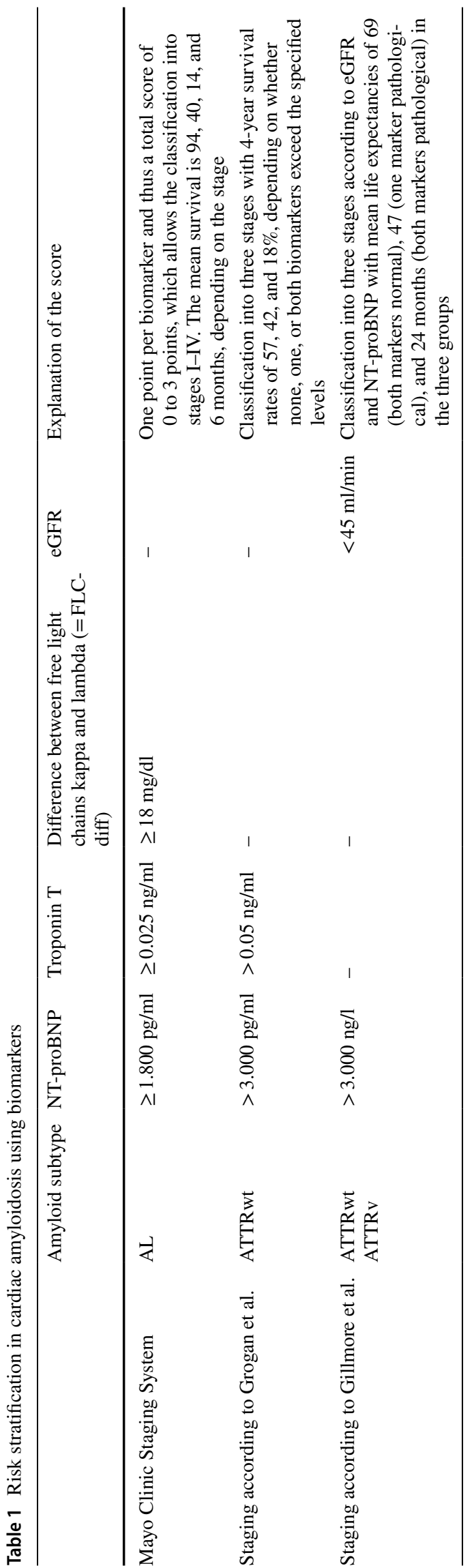


non-advanced AL amyloidosis who respond to therapy with haematological remission, median survival was reported to be over 5 years. However, in patients with advanced disease stages and without treatment, the disease usually leads to death after 6-12 months [18]. Therefore, early diagnosis has a significant impact on the survival rate.

\section{Clinical and cardiac manifestations of ATTRv and ATTRwt amyloidosis}

The phenotype of ATTR amyloidosis and also the age at first manifestation depend on the genotype, i.e., on the particular TTR gene mutation. Some mutations result in both neurological and cardiac symptoms, while others either lead to an almost exclusively neurological appearance, especially at a young age, or rarely to an isolated cardiac disease manifestation [25].

The most common phenotype, particularly in patients with ATTRwt amyloidosis, is the cardiac manifestation. The amyloid deposits can infiltrate all structures of the heart and, in addition to the ventricular and atrial wall, can also affect the conduction system, the heart valves, and the coronaries. Thus, the clinical spectrum of cardiovascular involvement is broad, ranging from asymptomatic courses, the occurrence of dizziness and syncope to the development of restrictive cardiomyopathy and progressive terminal heart failure. The cardiac biomarkers NT-proBNP and troponin T are often elevated, but not as pronounced as in patients with AL amyloidosis [25].

Patients with ATTRwt amyloidosis also show a relatively larger left-ventricular wall thickness compared to patients with other forms of amyloidosis [26]. ATTRwt amyloidosis is characterized by a late onset of the disease (usually after the 7th decade of life) and occurs most frequently in men. The heart is mainly affected in up to $90 \%$ of patients.
Initially, unspecific symptoms such as fatigue, performance slumps, and exertional dyspnoea appear as early symptoms of the disease [27]. During the course of the disease, the patients then present signs of heart failure (87\%) and cardiac arrhythmias (65\%) such as atrial fibrillation or AV blocks, but also ventricular arrhythmias [28, 29].

The primary site of extracardiac manifestation is the carpal tunnel. Carpal tunnel syndrome (CTS) occurs in up to $70 \%$ of patients with ATTRwt amyloidosis 5-10 years before cardiac manifestation. Furthermore, spinal canal stenosis may occur. An atraumatic biceps tendon rupture occurs in about $33 \%$ of patients. Findings such as an existing carpal tunnel syndrome (particularly bilaterally in men), neuropathic pain of unclear origin, orthostatic hypotension, and especially the evidence of unclear left-ventricular wall thickening after the 6th decade of life are indicative for the diagnosis of ATTR amyloidosis [30] (Table 2).

In particular, the presence of left-ventricular wall thickening which is not explained by arterial hypertension should always raise the suspicion of ATTRwt amyloidosis in older men [17]. Furthermore, in patients with calcifying aortic stenosis, ATTR amyloidosis was described in up to $32 \%$ of male patients aged $\geq 74$ [31]. Recent studies suggest a prevalence of $12-16 \%[32,33]$. The survival of these patients after interventional or surgical valve replacement is significantly worse when compared to patients without amyloidosis [33, 34].

The clinical manifestation of ATTRv amyloidosis depends on the mutation and other factors such as age at diagnosis, heredity pattern, gender, geographical conditions, and ethnicity [28]. The cardiac symptoms in patients with ATTRv amyloidosis are very similar to the symptoms of patients with ATTRwt, but the neurological manifestations can be more pronounced. Axonal sensorimotor polyneuropathy is a classic symptom of ATTRv amyloidosis. It usually begins with a disturbed sensitivity to pain and temperature in
Table 2 Signs for cardiac amyloidosis
Cardiac amyloidosis should be considered if, in addition to concentric LV wall thickening ( $\geq 12 \mathrm{~mm}$ - - in the absence of hypertensive heart disease — one of the following points is present

Age $>60$ years, symptoms of heart failure and still normal-sized ventricles

Low voltage or detection of an AV block in the resting ECG

Evidence of pericardial effusion, interatrial thickening, an echo hyperintense myocardial texture, a wall thickening of the RV, a valve thickening, or an "apical sparing"

Pathognomonic macroglossia with notches in the lateral parts of the tongue

Periorbital purpura (typically after minor injuries)

Atraumatic biceps tendon rupture

Carpal tunnel syndrome (mostly on both sides)

Sensorimotor polyneuropathy, neuropathic pain of unknown origin

Spinal stenosis

Autonomic dysfunction as well as orthostatic hypotension and erectile dysfunction

Vitreous opacity and pathognomonic pupillary changes 
the lower extremities with a proximally ascending pattern as the disease progresses. In addition, numbness and tingling as well as muscle weakness and walking disorders may occur. Carpal tunnel syndrome is also very common in patients with ATTRv amyloidosis. Autonomic dysfunctions such as orthostatic hypotension and erectile dysfunction as well as diarrhoea can also occur more frequently. With certain TTR gene mutations, vitreous opacities and pathognomonic pupil changes can also be detected. Involvement of the central nervous system is rare; leptomeningeal amyloidosis has, however, been described in some rare TTR mutations with a neurological clinical picture of dementia, stroke, ataxia, or spastic paralysis.

\section{Disease progression and prognosis of ATTRv and ATTRwt amyloidosis}

Patients with ATTR amyloidosis have a statistically better prognosis compared to patients with AL amyloidosis. However, the natural individual course of ATTR amyloidosis is difficult to assess due to the multiple factors influencing the disease and the heterogeneity of genotypes. In particular, the progression of neurological disease and cardiac manifestation are the leading causes of death in patients with ATTR amyloidosis. Polyneuropathy disability can be classified into three stages: free walking (stage I), dependent on a walking aid (stage II), and wheelchair bound (stage III).

Data on the prognosis of patients with cardiac ATTR amyloidosis depend on the initial individual stage of the disease and the population under consideration. The results of the "Transthyretin Amyloid Outcome Survey" (THAOS), which focused on ATTR patients in the USA, suggest a mortality rate of $>30 \%$ within 2 years for patients with ATTRwt, while for ATTRv with the p.Val142Ile mutation, even a mortality rate of $>40 \%$ within 2 years was observed [35]. The Transthyretin Amyloid Cohort Study (TRACS) also showed a marked difference in mortality between patients with ATTRwt and ATTRv (p.Val142Ile) (22\% vs. 79\% after an observation period of $\sim 15$ months) [36].

In patients with ATTRwt, the cardiac biomarkers NTproBNP and troponin $\mathrm{T}$ can also be used for risk stratification [37]. The staging system of the UK National Amyloidosis Centre provides prognostic information by measurement of the NT-proBNP concentration and the estimated glomerular filtration rate in patients with ATTRv as well as ATTRwt [38] (Table 1). Without treatment, every 6 months, the 6-min walking distance decreased by $\sim 26 \mathrm{~m}, \mathrm{NT}$-proBNP increased by $1816 \mathrm{pg} / \mathrm{ml}$, and left-ventricular ejection fraction deteriorated by $3.2 \%$. However, the number of patients examined in this study $(n=29)$ was very small. According to a somewhat larger study, the median life expectancy of ATTRwt is 47 months (after diagnosis) and the mortality rate is $64 \%$ after 5 years [39].

\section{Clinical and cardiac manifestation of AA amyloidosis}

Since AA amyloidosis primarily affects the kidneys, it usually manifests itself as renal insufficiency, often with proteinuria. At the time of the onset of clinical symptoms, there is usually already a manifest, advanced organ involvement. In earlier stages of the disease, only histological evidence is possible. A gastrointestinal involvement can often also be detected, but this typically remains asymptomatic for a long time. In the course of the disease, diarrhoea, gastrointestinal bleeding, and malabsorption disorders may occur. Liver, spleen, and adrenal gland involvement can often be detected histologically, but does not manifest itself clinically over a long period of time. Cardiac involvement in AA amyloidosis is considered rare and untypical $(<10 \%)[40,41]$; only one study was able to demonstrate a higher case rate in long-term observation of patients with rheumatological diseases (26\%) [42]. Since only case reports on cardiac AA amyloidosis have been available, little is known about clinical and morphological details.

\section{Disease progression and prognosis of AA amyloidosis}

Mean 5-year survival in most studies is about $40 \%$, only in one study, a mean survival time after the initial diagnosis of about 11 years was found [41]. However, it should be pointed out that there have been improvements in the therapy of the respective underlying disease, which might have a positive influence in the future. Predictors of poor outcome include elevated SAA concentration, significantly reduced renal function at the time of initial diagnosis, and cardiac involvement [42]. According to one study, the median survival is $\sim 6$ years for patients without heart involvement and $\sim 2$ years for patients with heart involvement [43].

\section{Diagnostic assessments for cardiac amyloidosis}

\section{Basic laboratory diagnostics}

Laboratory diagnostics can help to evaluate organ involvement and its extent in all forms of amyloidosis. Measuring of transaminases, gamma-GT, AP, and bilirubin for the assessment of liver function is oligligatory, and may indicate liver or cardiac involvement with right heart failure and congestion. Total protein and albumin levels should be tested. Since the kidneys are affected in several forms of amyloidosis, creatinine, urea, and the estimated GFR should be determined. The quantification of proteinuria and albuminuria (in particular in 24-h urine collection) 
is a diagnostic criterion for the presence of renal involvement in AL and AA amyloidosis. White blood cell differential, CRP, electrolytes, and coagulation (INR, PTT) should also be determined in patients with amyloidosis. In AA amyloidosis, SAA is the most important follow-up and activity parameter.

\section{Specific cardiac biomarkers}

The cardiac biomarkers troponin T and NT-proBNP are part of the diagnostic criteria for the presence of cardiac involvement in all forms of amyloidosis. Biomarkers in the normal range virtually exclude relevant cardiac involvement, while elevated biomarkers may indicate cardiac involvement, but are not specific for amyloidosis and must be interpreted in the context of cardiac imaging. Biomarkers are also used for risk stratification in various staging systems (Table 1; $[37,44])$.

Single studies have shown a prognostic value for transthyretin (prealbumin) as a serum marker in ATTRwt amyloidosis. Low serum transthyretin levels at the time of initial diagnosis are prognostically unfavourable [45]. For the estimation of the extent of cardiac involvement in AL amyloidosis, the Mayo-classification already mentioned [based on troponin T, NT-proBNP, and difference between the free light chains kappa and lambda (FLC-diff)] is primarily crucial (Table 1) [23].

\section{Specific laboratory diagnostics for suspected AL amyloidosis}

If light-chain amyloidosis is suspected, additional laboratory tests are necessary. First, an electrophoresis with determination of the M-gradient and immunofixation electrophoresis in serum and urine are performed, followed by quantitative determination of the free light-chain kappa and lambda in serum as well as the calculation of the ratio and the difference [46]. A kappa-lambda ratio of $<0.26$ indicates monoclonal lambda gammopathy, while a kappa-lambda ratio of $>1.65$ indicates monoclonal kappa gammopathy. In addition, a quantification of albumin and protein excretion as well as free light-chain excretion should be performed in the 24-h urine collection. A proteinuria of $>500 \mathrm{mg}$ per day (mainly albumin) suggests a renal involvement. The free light chains (including the ratio and difference) are also used to assess the response to therapy.

\section{Electrocardiography (resting and Holter ECG)}

The 12-lead resting ECG is part of every cardiac evaluation in patients with suspected cardiac amyloidosis as well as of every follow-up visit. Typical ECG changes such as low voltage only occur in about half of the patients. The low voltage contrasts with the thickening of the left ventricular wall, which is not due to hypertrophy of the cardiomyocytes, but is caused by amyloid deposits in the extracellular space. In early stages, low voltage is usually not detectable and unspecific. A true low voltage (QRS $<0.5 \mathrm{mV}$ in limb leads and $<1 \mathrm{mV}$ in chest wall leads) occurs in 50\% (AL) and 30\% (ATTR) of cases of cardiac amyloidosis [47].

Furthermore, cardiac amyloidosis often results in a prolongation of the QTc interval. For example, in patients with left-ventricular wall thickening, a QTc time extension of $>440$ ms with a simultaneous Sokolow-Lyon index of $<1.5 \mathrm{mV}$ (according to a smaller study) has a sensitivity of $85 \%$ and a specificity of $100 \%$ for detecting cardiac amyloidosis [48]. Other ECG signs include pseudo-infarct constellations and QRS broadenings.

In view of modern non-invasive imaging techniques, which allow both a more sensitive and more specific diagnosis of cardiac amyloidosis, the resting ECG plays a subordinate role.

In patients with cardiac amyloidosis, there is a general indication for a Holter ECG. If symptoms such as vertigo, syncope, and palpitations are also present, a (renewed) longterm ECG should be performed. AV blockages and bradycardias are rather common complications of cardiac amyloidosis leading to pacemaker or ICD implantation (depending on the extent of cardiac involvement). In addition to the AV block, the detection of (asymptomatic) atrial fibrillation is also important. Atrial fibrillation has been described in $5-18 \%$ of patients with ATTRv and $27-62 \%$ with ATTRwt amyloidosis [49] with the indication for oral anticoagulation. The significance of ventricular arrhythmias, on the other hand, is controversially discussed (see "Device therapy for cardiac amyloidosis" section).

\section{Tilt table test}

Tilting table test is rarely performed today and is often unavailable for patients with cardiac amyloidosis. In cardiac amyloidosis, orthostatic hypotension occurs with (secondary) autonomic dysfunction. The tilting table plays a defined role in the clarification of orthostatic dysfunction [50]. As a consequence, antihypertensive drugs and diuretics should be reduced in individual cases. However, a Holter ECG should be carried out first (if necessary also the implantation of an event recorder) to exclude bradycardic or tachycardic arrhythmias. 


\section{Echocardiography}

Transthoracic echocardiography is the primary and most widely available cardiological diagnostic imaging tool for patients with suspected cardiac amyloidosis. It is costeffective, universally available, radiation-free, and has no contraindications. It can also be easily performed as part of a family assessment in ATTRv.

Cardiac amyloidosis often appears in the late stages as restrictive cardiomyopathy with corresponding biatrial dilatation, diastolic dysfunction, and LV wall thickening (usually with an echogenic septal myocardium described as "granular sparkling" phenomenon). In the early stages, wall thickening is often not yet visible. An echocardiographic follow-up should also be carried out regularly (starting 10 years prior to the predicted age of onset and thereafter every year) in asymptomatic individuals who carry the mutation.

In addition to the quantitative description of wall thickening, the assessment of the size of the left atrium (biplane volumetry and indexing to the body surface; LA volume index) and of the diastolic function of the left heart is important. In addition to atrial enlargement, atrial amyloid deposits (thickening of the atrial walls) are also described, which further increase the risk for atrial fibrillation. Besides the transmitral E/A ratio, a tissue Doppler measurement should always be performed in the basal segments of the LV lateral and septal walls. In addition to the $\mathrm{E} / \mathrm{E}^{\prime}$ ratio, the pulmonary venous flow in the right upper pulmonary vein can often be displayed very well. For follow-up (under treatment), these parameters should be recorded.

Cardiac amyloidosis often shows a slight pericardial effusion, which, in most cases, is not hemodynamically relevant. In every echocardiography exam, it should be analysed in the subcostal plane: presence of compression of the right atrium. Cardiac amyloidosis only rarely leads to pericardial tamponade [51]. Complications of cardiac amyloidosis include wall thickening and diastolic dysfunction, as well as mitral regurgitation and post-capillary pulmonary hypertension. In some patients, LV outflow tract obstruction due to increased wall thickness was observed.

Left-ventricular ejection fraction (LVEF) is often only slightly reduced in early stages. In cardiac amyloidosis, measuring LVEF without knowledge of volumes and stroke volume is not a reliable parameter to assess systolic LV function; low-output hemodynamics may be present despite normal LVEF. LVEF is primarily a parameter of radial myocardial function and allows only little information about longitudinal shortening. As the loss in LV longitudinal shortening is compensated by increased radial thickening, the LVEF remains formally "normal" until late in the course of the disease.

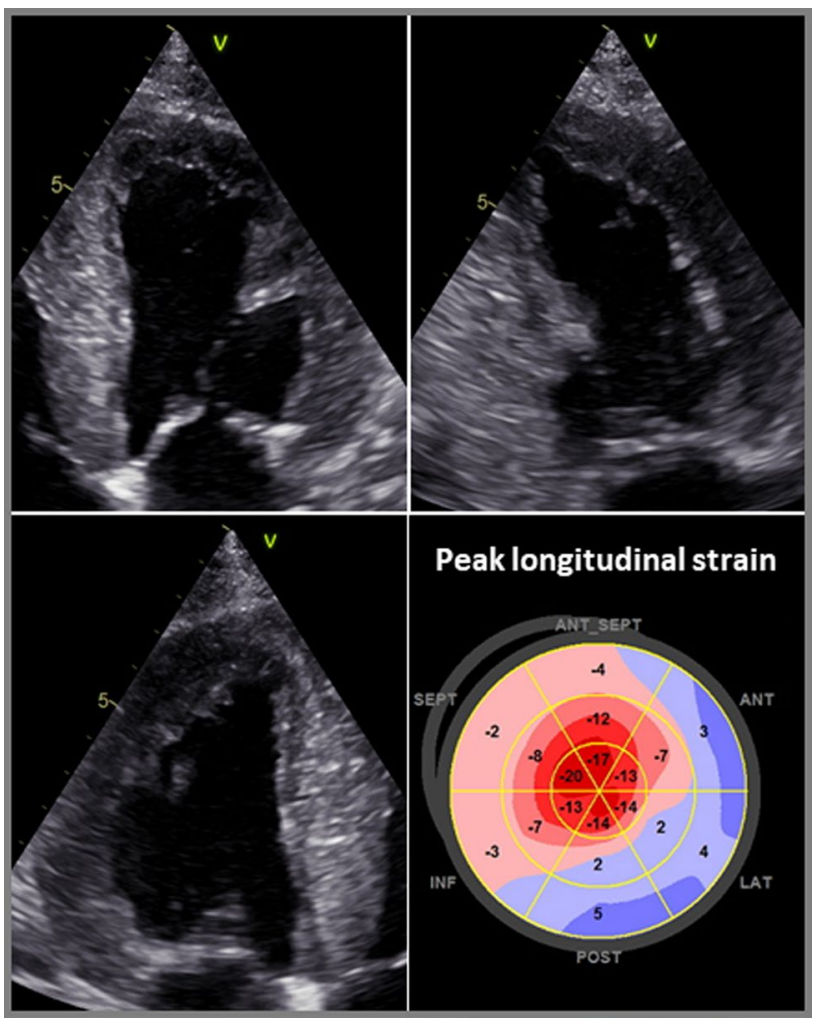

Fig. 1 Transthoracic echocardiography with measurement of longitudinal strain and detection of a characteristic "apical sparing" phenomenon in a patient with cardiac amyloidosis

Speckle tracking echocardiography ("deformation imaging") is a newer technique that should be used in all patients with suspected cardiac amyloidosis and is also recommended in current European (ESC) guidelines [52, 53]. The "deformation" is defined as the percentage shortening of a myocardial segment in relation to the initial length. In the 2D image, individual "speckles" (characteristic pixels) are tracked from image to image ("tracking"). From the movement of the "speckles" in a systole, the deformation (i.e., the percentage approximation of adjacent pixels during a systole) can be calculated. In addition to assessing the global strain ("global longitudinal strain" [GLS]), the regional myocardial function can also be assessed with lower intraand inter-observer variability.

In patients with cardiac amyloidosis, it is usually possible to determine longitudinal systolic function in all myocardial segments (GLS). These patients also typically exhibit the phenomenon of "apical sparing": this typical pattern shows a disturbance of the longitudinal strain in the basal segments with nearly normal longitudinal function of the apical segments (Fig. 1). According to some studies, "apical sparing" is specific and sensitive (sensitivity of $93 \%$ and specificity of $82 \%$ compared to controls and patients with other forms of LV wall thickening) [54]. It correlates with the scar extent in 
CMR imaging and is also prognostically significant [55], but cannot reliably distinguish between the different subforms of cardiac amyloidosis [56].

An important differential diagnosis of cardiac amyloidosis is the more common classical hypertrophic cardiomyopathy (HCM) —often caused by sarcomere protein mutations, in which an SAM phenomenon or an intraventricular obstruction is often detectable. Therefore, if the LV wall thickening is unclear, a Valsalva manoeuvre should always be carried out to exclude a dynamic outflow tract obstruction. Other differential diagnoses of amyloidosis include hypertensive heart disease, other storage diseases such as glycogenosis, hemochromatosis, or Fabry disease, which often involves wall thickening with prominent papillary muscles, although papillary muscle involvement can also occur in cardiac amyloidosis. Speckle tracking-based strain imaging can help in the differential diagnosis; the presence of "apical sparing" may indicate cardiac amyloidosis.

Especially, elderly patients with cardiac ATTRwt amyloidosis often have additional cardiac pathologies which have to be clarified by echo. The most important of these is the aortic valve stenosis. In the case of coincidence of cardiac amyloidosis and aortic valve stenosis, often, a "paradoxical low-flow low-gradient" aortic valve stenosis is present, which is often difficult to diagnose if classified only by the pressure gradient. This can only be diagnosed if the continuity method is also used and the stroke volume index is determined.

Cardiac amyloidosis is associated with increased occurrence of intracardiac thrombi. To rule out intraventricular thrombi, in addition to CMR imaging, contrast agent echocardiography is also useful [57]. In addition to transthoracic echocardiography, transoesophageal echocardiography is also an important method before cardioversion or initiation of rhythm control therapy for atrial fibrillation.

\section{Cardiovascular magnetic resonance imaging}

In recent years, cardiovascular magnetic resonance imaging (CMR) has gained considerable importance in the diagnosis of amyloidosis and its distinction from other cardiomyopathies. The following three strengths of the method are particularly important:

a) the exact analysis of heart function and anatomy,

b) the recording of myocardial infiltration using contrastenhanced images ("late gadolinium enhancement" [LGE]),

c) the recognition of diffuse changes in the myocardial extracellular space with specific mapping techniques.

\section{CMR-based analysis of heart anatomy}

The recording of global function, wall motion, myocardial mass, wall thickness, atrial size, and the interatrial septum is part of every routine CMR examination. The increased myocardial mass, significant thickening of the myocardium, enlargement of both atria, and thickening of the interatrial septum can be reliably detected in almost all patients. An advantage compared to echocardiography is the somewhat greater accuracy, especially in patients who cannot be examined well sonographically, the better differentiation of trabeculae and papillary muscles, the significantly sharper and higher-contrast epicardial differentiation, and the complete imaging of both atria. Global and regional strain analyses can be performed with, e.g., the feature tracking method using standard CMR (cine) images [58]. Beyond

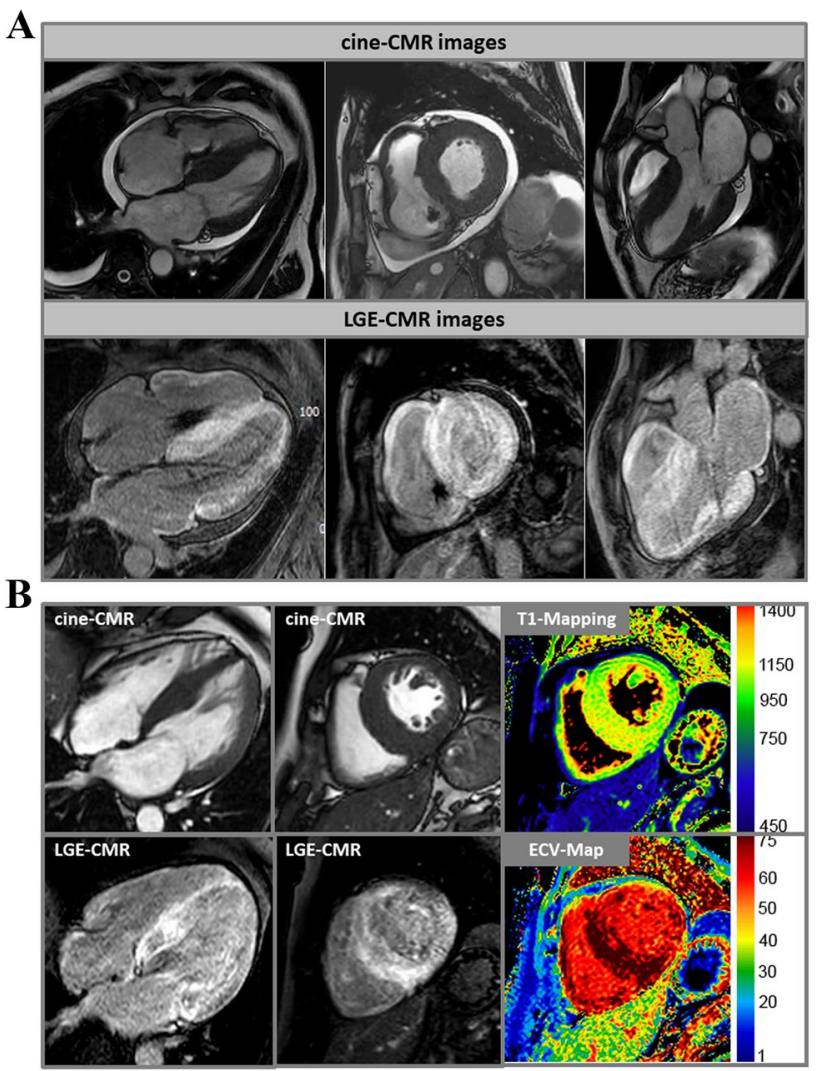

Fig. 2 a Cardiovascular magnetic resonance (CMR) images of a patient with cardiac amyloidosis and a DDD pacemaker. In the upper row, cine images are shown in a 4-chamber view, in the short axis, and in a 3-chamber view. In the lower series, the corresponding contrast images ("late gadolinium enhancement" [LGE]) are shown with evidence of diffuse LGE in all heart cavities and myocardial sections. b In addition to the cine and LGE images, T1 mapping and ECV (extracellular volume fraction) images are shown in the right column. Particularly in the basal septum, measured values of T1 (native) $=1300 \mathrm{~ms}$ and $\mathrm{ECV}=70 \%$ were significantly increased and characteristic for cardiac amyloidosis 
the improved visualization itself, CMR does not offer any diagnostic advantages in terms of merely anatomical visualization or functional evaluation.

\section{Detection of infiltration by contrast-enhanced CMR}

The increased interstitial volume in amyloidosis leads to a correspondingly increased volume of distribution for the extracellular gadolinium-containing contrast media (CM) commonly used in CMR [59, 60]. A few minutes after administration of CM, all infiltrated myocardial segments accumulate it and delimit themselves from unaffected or less affected heart segments. The diagnosis is more challenging if the respective myocardial damage is rather diffuse and quite homogeneous, as the LGE method is particularly good at visualizing regional differences, but global changes can appear pseudonormal. An early observation was therefore that, in amyloidosis, the signal intensities in the blood and myocardium rapidly converge, thus eliminating the usual contrast between blood and myocardium. Such a difficulty in achieving the usual contrasts in LGE imaging is a clear indication for the presence of a pronounced amyloidosis. In almost all cases, however, the entire heart muscle is not equally affected, so that regional differences with emphasis on the basal and subendocardial segments can also be recorded with the LGE method. LGE is not restricted to typical vascular territories or sharply defined as in case of myocardial infarction. Often diffuse and in part transmural changes can be seen (Fig. 2a). Both the right ventricle and the atrial walls may be affected. These typical and specific LGE patterns allow a clear identification of cardiac amyloidosis with sensitivities of $\sim 90 \%$ and specificities of $~ 90 \%$ [61, 62]. A high-quality CMR study with a characteristic LGE finding as shown in Fig. 2a is diagnostic of cardiac amyloidosis and similar to scintigraphy, the combination of such characteristic LGE findings with negative monoclonal protein studies will result in a high positive predictive value for the diagnosis of ATTR amyloidosis.

With the LGE technique, CMR thus offers a method that allows a direct view into the myocardium and also detects cardiac involvement in which the heart function is not measurably impaired or in which the wall thicknesses are still in normal or otherwise explicable ranges. In addition, the presence of global or transmural LGE is associated with a highly adverse prognosis (HR 2.93-5.4) and goes beyond the prognostic value of other markers (e.g., NT-proBNP or LVEF) [63, 64].

A frequently mentioned problem is the administration of gadolinium-containing $\mathrm{CM}$ in cases of impaired renal function. The often held opinion that these CM should not be given in patients with impaired renal function $(e G F R<30)$ has been relativized in recent years. If cyclic gadolinium chelates are chosen, the administration is usually possible even in cases of impaired renal function, provided that the indication has been strictly defined and the patient has been informed. However, the smallest possible dose (e.g., $0.0075-0.01 \mathrm{mmol} / \mathrm{kg}$ body weight) should always be aimed for.

\section{The detection of a diffuse change in extracellular space with mapping techniques}

With new methods, the magnetization of the heart muscle can be measured directly ("mapping"). Mapping techniques do not require regional differences, but show diffuse abnormalities by changing the absolute values [65]. Native T1 mapping does not require a contrast agent and provides a quantitative measure of the severity of the disease. Native T1 mapping can detect cardiac amyloidosis as accurately as $\mathrm{CM}$-assisted methods and shows significantly higher values for cardiac amyloidosis than in patients with hypertrophic cardiomyopathy, who also show an increase compared to heart-healthy or hypertensive patients [66-68]. Patients with $\mathrm{AL}$ amyloidosis usually have more pronounced changes than patients with ATTR amyloidosis (Fig. 2b). The increase in native $\mathrm{T} 1$ time for each subtype is correlated with the amount of amyloid deposition. Since T1 standard values may vary depending on the equipment and protocol, each MRI centre should define its own standard values, following the recommendations of the professional societies [69].

For both amyloid subtypes, the amount of native T1 time is closely linked to the prognosis. The significance of possible differences between native $\mathrm{T} 1$ time, which indicates changes in intercellular and interstitial space, and extracellular volume (ECV), which is determined by T1 mapping before and after $\mathrm{CM}$ administration and has a stronger emphasis on extracellular space, has not yet been conclusively clarified [70, 71]. However, the higher ECV values in ATTR compared to AL indicate pathophysiological differences at the cellular level between different types of amyloidosis. In a direct comparison of the hazard ratio values between ECV, LGE, and bone scintigraphy (based on the use of technetium $\left[{ }^{99 \mathrm{~m}} \mathrm{Tc}\right]$ phosphates), T1 and ECV data were prognostically superior to both LGE and scintigraphy [70]. So far, however, these studies are relatively small monocentric studies with a rather short observation period.

While scintigraphic methods only allow for the diagnosis of amyloidosis, CMR offers the great advantage that different aetiologies associated with LV wall thickening or hypertrophy can be clarified simultaneously and the exact underlying cause can usually be correctly assigned even in the case of a non-amyloidosis-associated LV wall thickening. Therefore, the current heart failure guidelines of the ESC recommend a CMR study for tissue characterization in patients with heart failure symptoms (I/C) [53]. Moreover, CMR is expected to play a central future role regarding 
non-invasive therapy monitoring in patients with cardiac amyloidosis receiving specific therapies [72, 73] due to its unique capabilities regarding non-invasive myocardial tissue characterization including depiction and quantification of amyloid load.

In summary, CMR offers an indispensable method for the detection of cardiac amyloidosis, both in the context of classical methods (LGE) and by extension to the new mapping procedures, and should be used early in cases of unclear left-ventricular hypertrophy.

\section{Nuclear imaging techniques}

Nuclear imaging approaches for the non-invasive diagnosis of cardiac amyloidosis currently include primarily scintigraphy and positron emission tomography (PET)-based procedures.

\section{Scintigraphy for the diagnosis of cardiac amyloidosis}

Current scintigraphic methods rely on the use of radionuclides based on ${ }^{99 \mathrm{~m}} \mathrm{Tc}$ phosphates. ${ }^{99 \mathrm{~m}} \mathrm{Tc}$ phosphates were initially developed for bone scintigraphy in the 1960s and later studied for myocardial infarction imaging [74]. In cardiology, they have experienced a renaissance in recent years in the context of cardiac amyloidosis diagnosis, although the first reports on this topic were already published in the early 1980s [75].
The ${ }^{99 \mathrm{~m}} \mathrm{Tc}$ phosphates currently in clinical use for cardiac amyloidosis diagnostics in Europe include ${ }^{99 \mathrm{~m}} \mathrm{Tc}-\mathrm{DPD}$ (3,3-diphosphono-1,2-propanodicarboxylate) and ${ }^{99 \mathrm{~m}} \mathrm{Tc}$ HMDP (hydroxymethylene-diphosphonate). By contrast, in the United States, ${ }^{99 \mathrm{~m}}$ Tc-PYP (pyrophosphate) is commonly used, while DPD is not approved [76-78]. The exact mechanism that leads to the increased affinity of these radionuclides to amyloid deposits in the heart has not yet been clarified in detail, but is suspected to be due to microcalcifications related to amyloid deposits. Also, the exact reason for the slightly higher affinity of these radionuclides for myocardial ATTR deposits compared to AL deposits is still unclear.

The applied amount of radionuclides or the whole procedure leads to a low average effective radiation dose of approx. 2-4 $\mathrm{mSv}$ per person and examination, which is less than the annual individual background radiation dose from natural sources [79]. Depending on the protocol, the total duration of the examination or stay of the patient from the first injection to the last image acquisition is up to 1-3 h, whereby planar scintigraphy is performed, followed by dedicated cardiac single-photon-emission-computedtomography (SPECT) imaging in case of positive planar imaging (Fig. 3). Of note, no specific patient preparation is warranted. In recent years, the following two approaches in particular have become established for image interpretation: (a) a semi-quantitative visual analysis using the so-called Perugini Score, a point scale of 0 to 3 , where $0=$ no cardiac

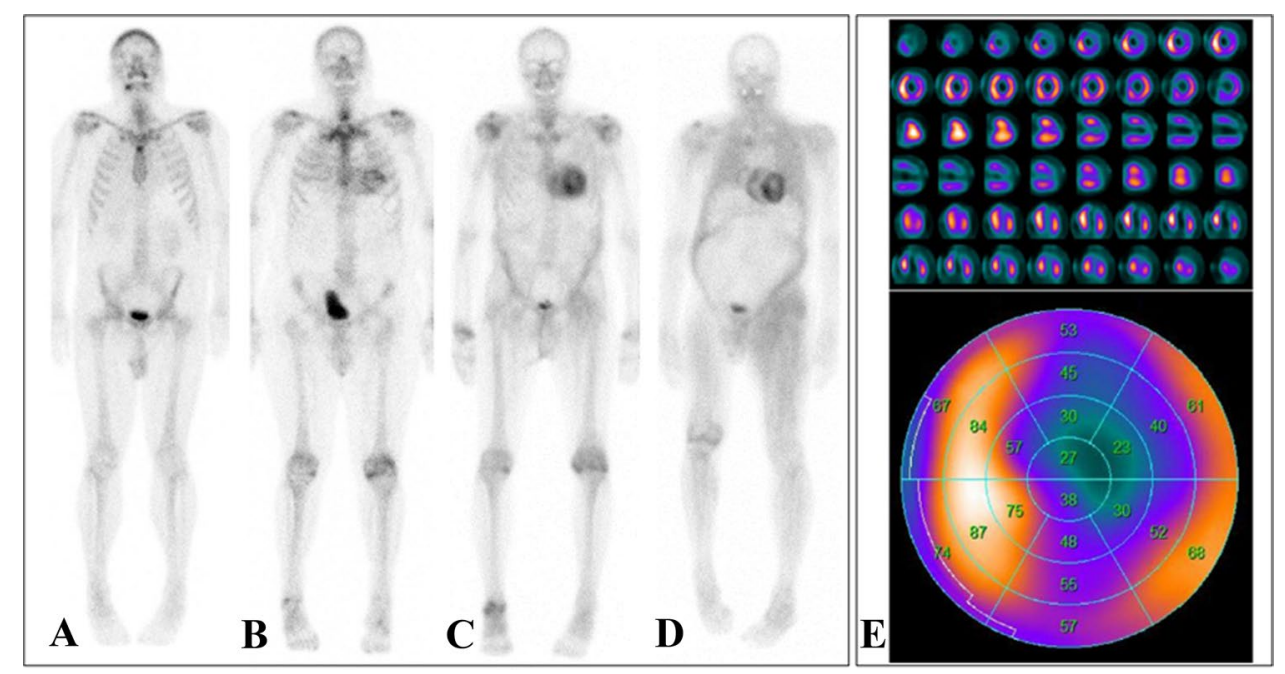

Fig. 3 Planar 99mTc-DPD scintigraphies of four different patients with varying degrees of cardiac radionuclide uptake: No cardiac uptake (Perugini score 0, a). Light cardiac uptake with preserved delineation of bone tissue (Perugini score 1, b). Strong cardiac uptake above that of bone tissue and increased soft-tissue uptake, particularly in the shoulder, abdominal wall, and gluteal region (Perugini score 2, c). Strong cardiac and soft-tissue uptake with no discernable bone-tissue uptake, suggesting diffuse amyloid soft-tissue infiltration
(Perugini score 3, d). In case of myocardial uptake on planar scintigraphy, SPECT imaging should be performed, which allows for a detailed assessment of radionuclide distribution within the left-ventricular myocardium (e, short axis, vertical long axis, and horizontal long axis slices at the top and the corresponding polar plot at the bottom; white/yellow indicates high uptake; blue/black indicates little or no uptake) 
uptake and normal bone uptake, $1=$ mild cardiac uptake less than bone uptake, $2=$ moderate cardiac uptake and relatively equal bone uptake, and $3=$ high cardiac uptake and only mild or absent bone uptake [78]; (b) a semi-quantitative analysis that determines the ratio of tracer uptake between the heart (H) and the contralateral half of the lung (CL), referred to as the H/CL ratio [80] (Fig. 3).

A number of single-center studies have confirmed the high diagnostic accuracy of ${ }^{99 \mathrm{~m}} \mathrm{Tc}-\mathrm{DPD},{ }^{99 \mathrm{~m}} \mathrm{Tc}-\mathrm{HMDP}$ and ${ }^{99 m}$ Tc-PYP, yielding a sensitivity and specificity of $>90 \%$ [78, 81-84]. In line, a recent meta-analysis comprising 529 patients has reported a pooled sensitivity and specificity of 92\% (95\%-CI: 89-95\%) and 95\% (95\%-CI: 77-99\%), respectively [85]. Importantly, however, $99 \mathrm{mTc}$ phosphates are not specific to ATTR-related amyloid deposits but also exhibit increased focal uptake in a substantial proportion of patients (up to $20 \%$, but not all) with AL amyloidosis. Exclusion of AL amyloidosis by means of proving the absence of monoclonal proteins in serum and urine electrophoresis is therefore mandatory and confers the very high specificity of scintigraphy for the detection of cardiac ATTR-amyloid. A large international collaboration by Gillmore et al. [86] retrospectively evaluated the diagnostic performance of nuclear imaging in over 1200 patients referred for evaluation of suspected cardiac amyloidosis. Disregarding some methodological issues, the authors concluded that the collective findings of a Perugini score $\geq 2$ on planar scintigraphy along with the absence of monoclonal gammopathy on serum and urine electrophoresis yield a specificity and positive predictive value of $100 \%$ for ATTR cardiac amyloidosis, allowing confirmation of the diagnosis without the need for endomyocardial biopsy. Interestingly, however, the specificity of scans with a Perugini score $\geq 2$ was only $91 \%$ in the absence of exclusion of monoclonal protein. This underlines the necessity to exclude AL cardiac amyloidosis by immunofixation studies concurrently with bone-tracer imaging.

The main advantages of scintigraphy are its relatively low costs, its established reimbursement in Germany, the wider availability compared to $\mathrm{CMR}$, and the independence of image quality from patient-specific factors. Hence, scintigraphy is a highly valuable diagnostic tool for screening patients with suspected cardiac ATTR amyloidosis and current international guidelines recommend its use early in case of clinical symptoms, ECG, echocardiography, and/or CMR findings suspicious for cardiac amyloidosis [87, 88]. However, as outlined by Kittleson et al. recently [89], the test performance of scintigraphy in populations with lower disease prevalence is unknown and the aforementioned positive predictive value of scintigraphy (with the absence of monoclonal gammopathy) of $100 \%$ for ATTR is questioned by some recent reports that illustrate possible causes of falsepositive 99mTc-PYP scans [90, 91]. In addition, first larger studies with a direct comparison of scintigraphic ${ }^{99 \mathrm{~m}} \mathrm{Tc}-\mathrm{DPD}$ images with a CMR-based determination of ECV in the same patients suggest that there is a prognostic significance for the more precise CMR-based ECV [70].

With the recent introduction of therapeutic options, a need for diagnostic modalities allowing for treatment monitoring did arise. Therefore, means of quantifying disease burden become a requirement for future applications of imaging. Planar scintigraphy and SPECT imaging, however, inherently lack the possibility of quantification. By contrast, CMR-based determination of, e.g., the myocardial ECV in patients with proven cardiac amyloidosis may evolve as a valuable tool for estimating the extent of amyloid (including the differentiation of degrees of severity or the monitoring of progression over time) [92]. Alternatively, positron emission tomography (PET) may play an important future role, as it inherently provides quantitative measures of radionuclide uptake, potentially allowing for accurate assessment of the treatment response similar to that established for follow-up of inflammatory myocardial disease (e.g., cardiac sarcoidosis).

\section{PET for the diagnosis of cardiac amyloidosis}

Due to the limited number of (mostly retrospective) studies with small case numbers, it is not yet possible to make reliable statements on the use of promising positron emission tomography (PET) for the diagnosis of cardiac amyloidosis [93]. In previous PET studies, the following radionuclides have been used: ${ }^{11} \mathrm{C}$-Pittsburgh Compound (PIB), ${ }^{18} \mathrm{~F}$-florbetaben, and ${ }^{18} \mathrm{~F}$-florbetapir [94-96]. These radionuclides were primarily developed to detect $\beta$ amyloid plaques in the brains of Alzheimer's patients and appear to allow detection of both ATTR and AL deposits in the heart. While ${ }^{18} \mathrm{~F}^{-}$based radionuclides have a relatively long half-life of $>100 \mathrm{~min}$ and, therefore, do not necessarily need to be produced on site, PIB has a relatively short half-life and, therefore, needs to be used quickly after production. Dependent on tracer and technique, the average radiation exposure of amyloidtargeted PET ranges from 1.5 to $7 \mathrm{mSv}$ per patient and examination [97, 98]. For these radionuclides, diagnostic sensitivities of $87-100 \%$ and specificities of $83-100 \%$ for the diagnosis of cardiac amyloidosis have been determined in smaller monocentric studies [93]. Future studies are yet required to assess the potential of PET to differentiate between cardiac ATTR and AL amyloidosis. The strongest potential may, however, be in the quantitative nature, enabling therapy monitoring. Of note, a recent study using PIB-PET confirmed that the quantitative myocardial signal reflects the amount of amyloid deposit and is an independent predictor of adverse outcome in AL [99]. 


\section{Endomyocardial biopsy (EMB)}

Today, endomyocardial biopsy (EMB) still remains the gold standard for the diagnosis of many non-ischemic heart diseases, such as (viral) myocarditis, cardiac storage diseases, and also for infiltrative or restrictive cardiomyopathies such as cardiac amyloidosis. EMB can therefore result in a therapeutic decision with prognostic relevance in cardiomyopathies, e.g., in inflammatory diseases such as cardiac sarcoidosis or (giant cell) myocarditis. In each individual case, however, the advantages of EMB in terms of diagnosis and therapy implementation must be properly evaluated and weighed against other aspects, such as the sufficient availability of non-invasive information using modern imaging techniques or the potential complication risks of invasive EMB. The specific local conditions and experiences on site as well as the age of the patients should be taken into account.

Larger multi-centre studies have shown that the risk of complications of EMB for both left-ventricular (LV) and right-ventricular (RV) biopsy is low $(<1.0 \%)$ in experienced centres with high case numbers, and that serious or permanent complications are rare $[100,101]$.

Another important limitation of EMB procedures in previous studies was often the so-called "sampling error": since (viral) myocarditis, for example, has a rather irregular spotlike pattern and the focus of inflammation is often located more subepicardially; the subendocardial EMB sample may be histopathologically negative, even though myocarditis is present. In the case of cardiac amyloidosis, on the other hand, there is a more diffuse distribution of amyloid deposits (particularly in the advanced stage), especially in the basal layers of the LV, which, in most cases, also extends from subendocardial to intramural. In this respect, the risk of a "sampling error" of the subendocardial collection of an EMB in case of cardiac amyloidosis is lower than in case of myocarditis. Collection of at least $5 \mathrm{LV}$ or RV samples from the target region (if possible previously defined by imaging) is recommended for cardiac amyloidosis [102].

Cardiac amyloidosis often involves both the LV and the $\mathrm{RV}$, and the amyloid deposits are particularly pronounced in the septum as well as in the area of the free LV lateral wall. Since the septum can be reached particularly well with an RV biopsy and the LV lateral wall with an LV biopsy, both $\mathrm{RV}$ and LV biopsy are in principle suitable for the bioptic diagnosis of cardiac amyloidosis (Fig. 4) [103]. In individual cases, the availability of data from previous imaging (e.g., LGE distribution pattern in CMR; orientation of the septum in the transverse plane) is helpful, as this allows to better define in advance the target region for the biopsy as well as the methodological procedure and to reduce potential risks (e.g., unintentional puncture of the free RV wall).

The biopsy-proven extent of amyloidosis is of crucial therapeutic importance, particularly in AL amyloidosis: a bioptically quantified amyloid load of $>20 \%$ (of the EMB sample considered) is associated with a lower probability of a therapeutic benefit from consecutive chemotherapy [104]. For both ATTR and AL amyloidosis, there is evidence that a
Fig. 4 Schematic illustration of the target regions of endomyocardial biopsy of the right (RV) or left ventricle (LV). While in case of RV biopsy, the rightventricular parts of the septum can be reached very well, an LV biopsy allows sampling of tissue in the area of the left-ventricular lateral or inferolateral wall easily. In advanced cardiac amyloidosis, the risk for a sampling error is quite low for both RV and LV biopsy due to diffuse amyloid deposits

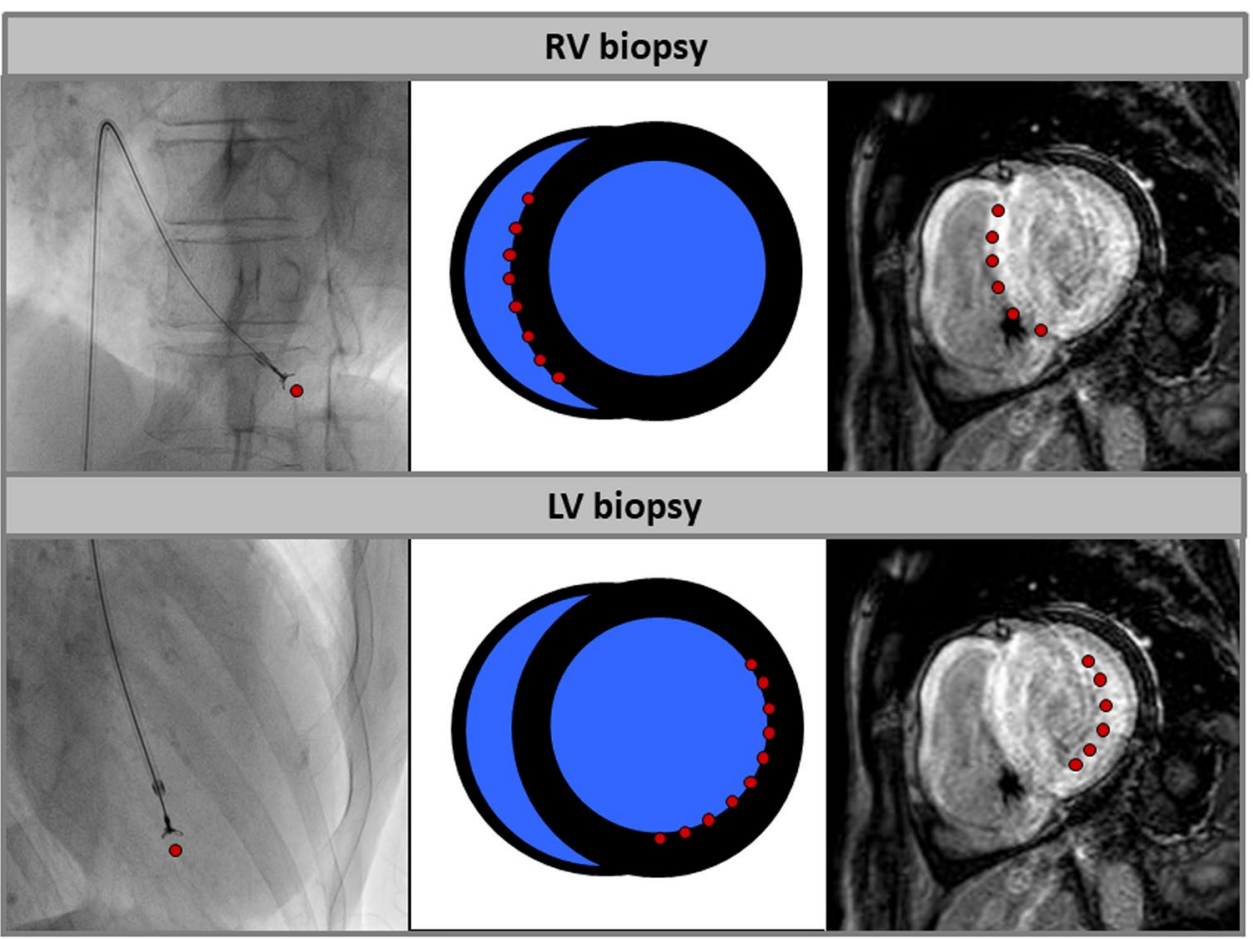


relatively high myocardial amyloid load is associated with a worse prognosis [105]. However, since (a) amyloid deposits in the myocardium can occur both in an irregular nodular fashion and diffusely interstitially and (b) a standardized procedure for the quantification of the amyloid load in EMB samples of cardiac amyloidosis is still lacking, valid and clinically usable data on the significance of the extent of amyloid, oedema, or inflammation in the EMB sample are not yet available.
In the current heart failure guidelines of the ESC, an EMB in patients with progressive heart failure symptoms is primarily recommended if (a) it is necessary to confirm the diagnosis and (b) has direct therapeutic consequences (strength of recommendation $\mathrm{IIa} / \mathrm{C}$ ) [53]. In general, these conditions currently apply in cases of suspected cardiac amyloidosis. Especially with regard to the increasing availability of new drug therapies, bioptic diagnosis including exact differentiation of cardiac amyloidosis (e.g., by
Fig. 5 a Electron microscopic detection of amyloid protein fibrils (in an endomyocardial tissue sample) that show a characteristic structure of antiparallel beta strands with a diameter of 7-13 nm. b Histopathological studies including immunohistochemical stainings allow an accurate classification of the amyloid subtype at an early stage of disease. c Typically, in cardiac AL amyloidosis, a rather reticular pattern is found, whereas in ATTR amyloidosis, focal amyloid deposits predominate
A

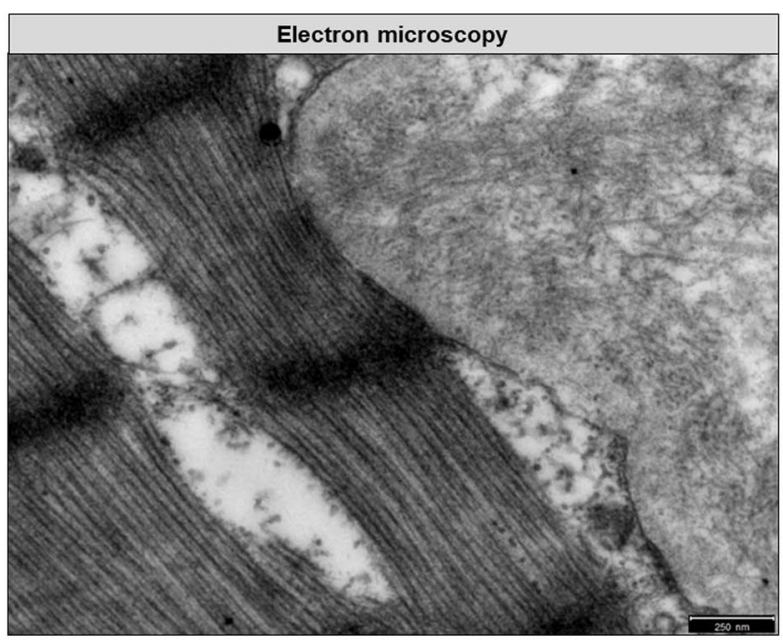

B

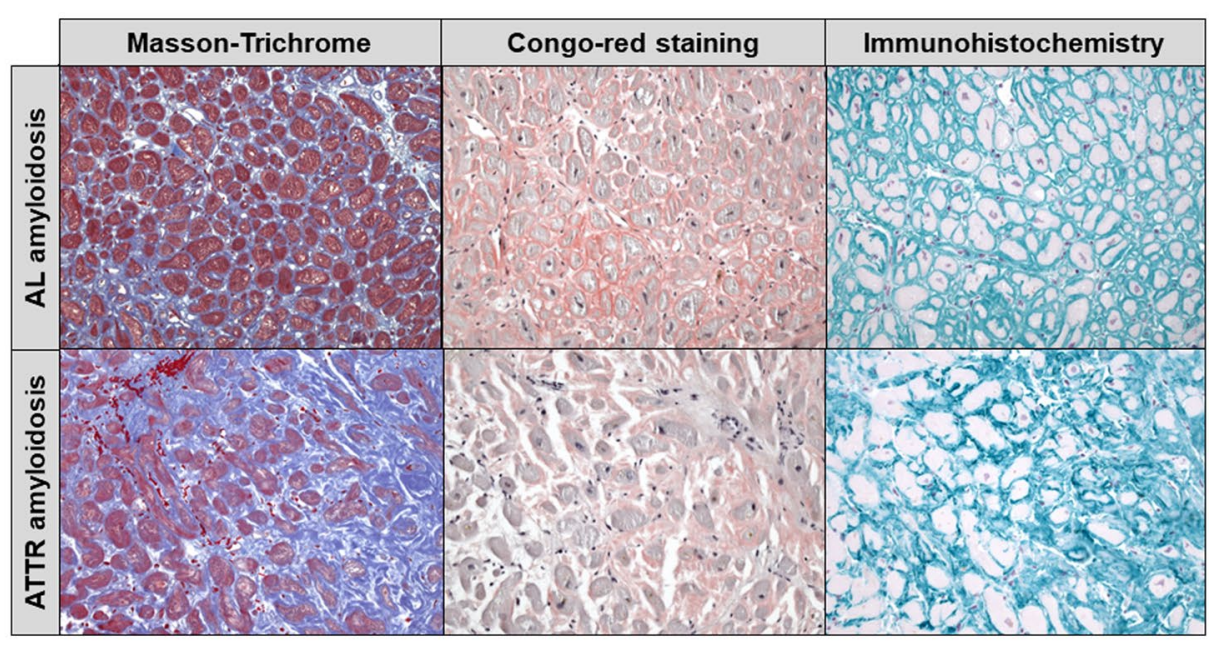

C

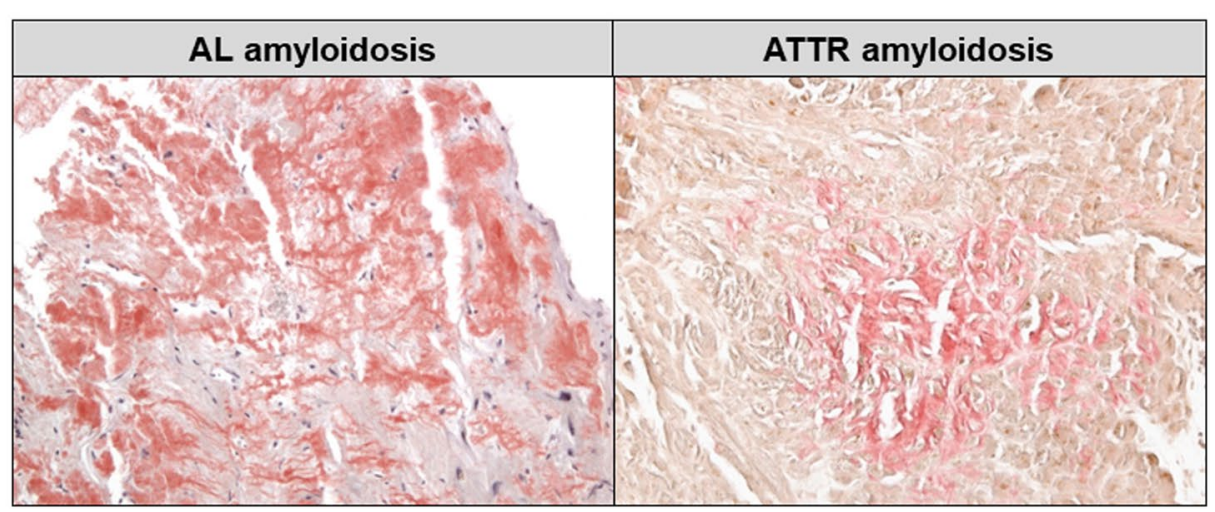


immunohistochemical staining) will continue to play a decisive role for the time being.

\section{Extracardiac biopsy}

Extracardiac tissue biopsy is used to confirm the diagnosis of systemic amyloidosis, especially in the case of non-cardiac involvement. The diagnostic certainty of non-cardiac sampling depends on the type of amyloidosis and the tissue examined [27]. Sampling from an affected organ is essential, as the sensitivity of the tissue sample for amyloid detection is highest. In a study with 286 patients with cardiac ATTR amyloidosis, only $73 \%$ of the patients showed positive amyloid detection in an extracardiac biopsy [106]. In patients with ATTR amyloidosis, on the other hand, confirmation of the diagnosis using non-cardiac biopsies was only possible in about $30 \%$ of cases. AL amyloidosis is most frequently detected in extracardiac biopsies, followed by ATTRv amyloidosis, whereas the sensitivity for the detection of ATTRwt amyloidosis is low in extracardiac biopsy. Therefore, if cardiac amyloidosis is suspected, it is recommended to perform amyloidosis diagnostics on endomyocardial biopsies, which have a significantly higher sensitivity if there is no unequivocal diagnosis made from non-invasive testing. More detailed explanations on extracardiac diagnostics can be found in the attachment (additional material online: Attachment Extracardiac Biopsy).

\section{Histological examination of biopsies for suspected amyloidosis}

All amyloid deposits consist of protein fibrils that have a remarkably similar structure of antiparallel beta strands (more rarely parallel beta strands) with a diameter of 7-13 nm, which can be easily identified by electron microscopy (EM) in the myocardium (Fig. 5a). Recently, EM studies revealed that pathological fibrils from brain and heart exhibit morphological homogeneity and that the physiological milieu is the key determinant of amyloid fibril strains [107]. Formalin-fixed and paraffin-embedded (FFPE) tissue samples of the left ventricle or right ventricle are routinely used for the diagnosis of cardiac amyloidosis. Amyloid is detected by Congo red staining, which shows a typical apple-green, but partly also yellow-orange birefringence when viewed under polarized light. The correct Congo red staining is also critical in heart biopsies, as these can easily be overstained and the result is then interpreted as false positive.

Crucial for the therapy is the accurate classification of the amyloid, which can be reliably performed already at an early stage of the disease with high sensitivity by immunohistochemical (IHC) staining in FFPE EMB. Reliable antibodies are used for the detection of ATTR, AL, and AA amyloidosis, which account for more than $99 \%$ of cardiac amyloidoses (Fig. 5b). Typically, in cardiac $\mathrm{AL}$ amyloidosis, a rather reticular/pericellular pattern is found, whereas in ATTR focal amyloid deposits predominate ([104]; Fig. 5c). In some designated laboratories, additional antibodies are available which can be used to identify rare cardiac amyloidoses (e.g., apolipoprotein AI, Lect2, and $\beta 2$-microglobulin).

For the immunohistochemical differentiation of amyloidoses, it must be taken into account that the incidence of monoclonal gammopathy of unclear significance (MGUS) is high $(5.3 \%$ in $>70$ years old, $7.5 \%$ in $>85$ years old) [108], $25 \%$ of patients with ATTRv or ATTRwt amyloidosis have in parallel MGUS, and $10 \%$ of systemic amyloidoses are misdiagnosed with regard to the form of amyloidosis [109].

For the identification of amyloid, there are further stains as thioflavin $\mathrm{T}$ or $\mathrm{S}$, but the fluorescence microscopy required for this is rarely available. Furthermore, staining with thioflavin is not entirely specific for amyloid. In recent years, luminescent conjugated polythiophenes (LCP) and oligothiophenes (LCO) have been developed for the histological diagnosis of amyloid, including h-FTAA ("heptamer formyl thiophene acetic acid"). The application of laser dissection mass spectrometry LDMS or mass spectrometry-based quantification by isotope-labeled cellfree products (MS-QBIC) in FFPE EMB might be useful for the classification of systemic amyloidoses.

\section{Genetic testing for suspected ATTR amyloidosis}

ATTR amyloidosis is the most common familial form of amyloidosis and is caused mainly by non-synonymous mutations in the transthyretin gene (TTR; Chr. 18q12.1; 147 amino acids), which lead to instability of the protein's tetrameric structure. There are now more than 100 TTR gene mutations known, of which about $40 \%$ are associated with systemic ATTR and 45\% ATTRv and neurological manifestation. The transmission of mutations is autosomal dominant, with variable penetrance and clinical manifestation. A familial occurrence of amyloidosis-related disease signs might be indicative of an inherited form.

Non-genetic forms of amyloidosis with cardiac involvement are, for example, the ATTRwt amyloidosis (criterion: exclusion of a TTR gene mutation) and ANF-associated amyloidoses (AANF), which manifest primarily as atrial cardiomyopathy. Other hereditary amyloidoses include genes for the apolipoprotein A-2 (APOA2; amyloidosis subform: AApoA2), for the $\alpha$ fibrinogen (FGA), the gelsolin (GSN, "finnish amyloidosis"; amyloidosis subform: AGel), lysozyme (LYZ; ALys), cystatin C (CYS3; ACys), or the " $\beta$ precursor protein" $(\mathrm{A} \beta)$, but do not show any cardiac manifestation. 


\section{Recommendations for genetic testing in cardiac amyloidosis}

- In an index patient with cardiac, but unclassified ATTR amyloidosis (after biopsy examination), a molecular genetic diagnosis for further differentiation (ATTRwt vs. ATTRv) with sequencing of the TTR gene should be performed.

- In selected cases, a genetic analysis of the TTR gene might be performed if clinical findings and in particular non-invasive imaging (echocardiography, CMR) suggest a strong suspicion of cardiac amyloidosis or otherwise unexplained LV hypertrophy; laboratory tests should have ruled out monoclonal gammopathy.

- In selected cases, an extended genetic diagnosis of further amyloidosis genes (e.g., if AApoA1 is suspected) may also be considered.

- Biologically related first-degree relatives of an index patient with a proven hereditary cardiac amyloidosis should be examined for signs of cardiac amyloidosis regardless of symptoms, and should undergo a mutation cascade screening to determine the mutation carrier sta- tus (after respective genetic counselling according to the current national gene diagnostic law).

\section{Correlations between genotype and cardiac phenotype in ATTRv}

A regional accumulation of ATTR amyloidosis is found in Japan, Sweden, and Portugal, particularly of ATTRv with neurological manifestation with a specific and most common TTR gene mutation (p.Val50Met). The age peak varies regionally ("early onset" in Portugal and Japan: 30-40 years; "late onset" in Sweden: 50-60 years). In the case of organ involvement, the polyneuropathy dominates (cardiac involvement usually only manifests itself later in life), although the gastrointestinal tract and the eyes may also be affected.

TTR gene mutations with primary cardiac involvement include the amino acid exchange mutations p.Val40Ile, p.Thr649Ala, p.Thr80Ala, p.Ile88Leu, p.Leu131Met, and p.Val142Ile (4\% for African Americans). In a large study, it was shown that both advancing age of onset of the disease and lower mean arterial blood pressure were independently

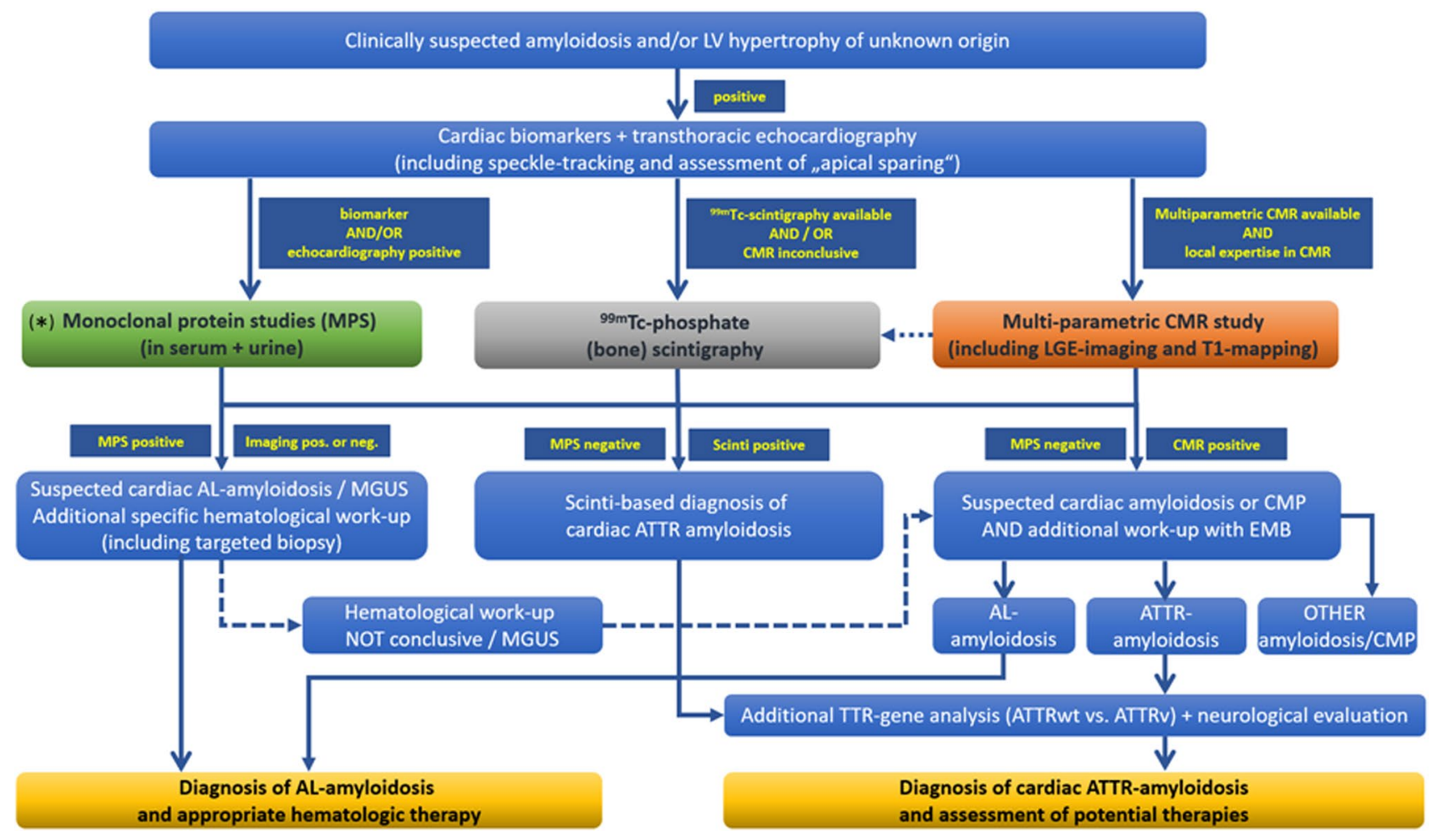

Fig. 6 Recommended path for the diagnostic work-up of suspected cardiac amyloidosis. The main objective of this diagnostic path is to detect the presence of cardiac amyloidosis as reliably and early as possible, to characterize the extent of cardiac amyloidosis as precisely as possible, to reliably identify the underlying type of amyloidosis, and to subsequently enable targeted treatment (including the possibility of monitoring the success of therapy). * =If cardiac amyloidosis is suspected, further clarification by means of free light chains and immunofixation for the determination of light chains is recommended promptly and without waiting for additional imaging results 
associated with a higher mortality compared to a specific, non-synonymous gene mutation [35].

A significant prognostic difference exists between AL and ATTR patients. In addition, smaller studies indicate that, within the ATTR group, certain forms of ATTRv (e.g., p.Thr80Ala or p.Val142Ile) have been associated with a particularly poor prognosis $[36,110]$.

\section{Diagnostic pathway for cardiac amyloidosis}

In view of the data available to date on the various noninvasive imaging procedures and invasive endomyocardial biopsy, we recommend-in addition to the laboratory tests described above- the diagnostic path outlined in Fig. 6. The main objective of this diagnostic path is (i) to detect the presence of cardiac amyloidosis as reliably and early as possible, (ii) to characterize the extent of cardiac amyloidosis as precisely as possible, (iii) to reliably identify the underlying type of amyloidosis, and (iv) to subsequently enable targeted treatment (including the possibility of monitoring the success of therapy). The implementation of this diagnostic path is of course dependent on the available options on site as well as local expertise and experience.

If cardiac amyloidosis is suspected, further clarification by means of free light chains and immunofixation (from serum and $24 \mathrm{~h}$ urine) for the determination of light chains as well as CMR and/or ${ }^{99 \mathrm{~m}} \mathrm{Tc}$-phosphate scintigraphy is recommended promptly - if possible in a specialized amyloidosis centre. Since AL amyloidosis progresses rapidly and the patient's prognosis depends on the extent of cardiac involvement and immediate initiation of therapy, an immunofixation consisting of serum and 24-h urine collection should be initiated immediately on site. This will allow for a timely presentation to a hematology service in case of detection of a monoclonal gammopathy.

Importantly, the decision whether to perform a multi-parametric CMR study and/or a ${ }^{99 \mathrm{~m}} \mathrm{Tc}$-phosphate scintigraphy for further non-invasive work-up of suspected cardiac amyloidosis depends (amongst others) on (a) local availability and (b) respective imaging expertise. Hence, the diagnostic path outlined in Fig. 6 needs to consider local peculiarities. The same is true regarding invasive sampling of EMBs.

It remains to be seen to what extent non-invasive imaging procedures will not only allow for the diagnosis of cardiac amyloidosis in the future, but also a reliable differentiation of different types of amyloidosis, thereby reducing the need for invasive EMB. In individual cases, an invasive EMB can be dispensed (especially in very old patients with severe frailty precluding invasive diagnostics) if (a) unequivocal echocardiographic, CMR, or scintigraphic findings of cardiac amyloidosis are available, (b) the presence of monoclonal bands has been ruled out, and/ or c) a specific drug therapy is not considered. In addition, even in younger patients ( $<50$ years $)$ with a family history and/or a symptom complex that clearly indicates systemic ATTRv amyloidosis, an EMB can be omitted for the time being and a genetic examination \pm extracardiac biopsy can be performed. Importantly, future studies need to further clarify whether an imaging-based diagnosis of cardiac amyloidosis-without a histopathological proof of amyloidosis-is appropriate and sufficient prior to initiating specific, however expensive, therapies (e.g., in case of ATTRwt).

However, due to the higher significance, EMB is still primarily recommended if cardiac amyloidosis is suspected. If an EMB is not technically possible, not desired by the patient, or not purposeful, an extracardiac biopsy (especially FAB) can be considered-despite a significantly lower sensitivity.

In asymptomatic ATTRv mutation carriers, a comprehensive screening for cardiac manifestation comprising ECG, serum biomarkers, and echocardiography is suggested to be performed 10 years prior to the predicted age of onset and annually thereafter [111]. The initial assessment may also include CMR or ${ }^{99 \mathrm{~m}} \mathrm{Tc}$-phosphate scintigraphy_depending on the underlying TTR gene mutation.

\section{Treatment of cardiac amyloidosis}

\section{General treatment recommendations}

In principle, the same general treatment recommendations apply to patients with symptomatic cardiac AL and ATTR amyloidosis as for patients with heart failure (HFrEF or HFpEF) due to other causes [53]. In amyloidosis patients, however, it should be noted that even low doses of $\beta$ lockers or ACE inhibitors can lead to symptomatic hypotension and are often not well tolerated. Since patients with cardiac amyloidosis also have a limited coronary perfusion reserve, a $\beta$-blocker therapy with consecutive reduction of the heart rate can lead to a drop in cardiac output with clinical deterioration of the state of compensation. Therefore, heart failure therapy in patients with cardiac amyloidosis is primarily based on the correct titration of diuretics.

There are currently insufficient data on the use of sacubitril/valsartan in patients with cardiac amyloidosis. A possible acceleration of amyloid deposition by sacubitril/valsartan was discussed.

Older case studies indicate that calcium channel blockers (in particular nondihydropyridine calcium channel blockers) or digitalis preparations should be used with restraint or caution in patients with cardiac amyloidosis, as diastolic dysfunction may further deteriorate and heart block may occur [112]. 


\section{Specific drug therapies for AL amyloidosis}

The timely treatment of underlying plasma cell dyscrasia or clonal B-cell disease is the main focus of AL amyloidosis therapy. The treatment regimes used are similar to those used for multiple myeloma. There is no specific therapy for cardiac involvement, but the degree of cardiac involvement often limits the choice of chemotherapy. The choice of therapy is based on dedicated risk stratification and includes criteria such as age, Karnofsky index, number of organs involved, and extent of organ involvement (kidney function, cardiac stage according to Mayo staging system, and NYHA stage) [113]. If the patients are suitable, a high-dose melphalan therapy followed by autologous stem cell transplantation is aimed for [114]. For patients in advanced stages, conventional therapies such as melphalan/dexamethasone or various combination therapies with the proteasome inhibitor bortezomib (or ixazomib) or immunomodulators are used $[115,116]$.

The duration of therapy depends on the response according to established international response criteria [117]; therefore, close monitoring of the activity parameters is necessary every 2-3 cycles. If the patient does not respond to primary therapy, an immediate switch to second-line therapy is mandatory. The treatment scheme should be determined in a specialised centre (also to allow inclusion in studies). As a non-drug therapy option, heart transplantation may be considered in selected cases to enable patients to receive high-dose chemotherapy and autologous stem cell transplant afterwards.

\section{Specific drug therapies for cardiac ATTR amyloidosis}

In the field of ATTRv amyloidosis, several different drugbased treatment strategies have been developed in recent years. With the aim of slowing down the progress of the disease, drugs that either stabilize the transthyretin tetramer or suppress the synthesis of the protein in the liver were tested in clinical trials. In Europe, three drugs are currently approved for the treatment of ATTRv polyneuropathy, and one drug was approved for therapy of amyloid cardiomyopathy (hereditary and wild-type) recently.

Since 2011, the TTR stabilizer Tafamidis-meglumin $\left(\right.$ Vyndaqel $^{\circledR}$ ) has been approved in Europe for the treatment of stage 1 amyloid polyneuropathy. In the first quarter of 2020, the European Medicines Agency (EMA) approved Tafamidis for treatment of amyloid cardiomyopathy based on the data from the recently published phase III clinical trial (ATTR-ACT). In this study, the safety and efficacy of Tafamidis ( $20 \mathrm{mg}$ vs. $80 \mathrm{mg}$ vs. placebo) was evaluated over 30 months in a cohort of patients with amyloid cardiomyopathy (106 ATTRv and 335 ATTRwt patients) [118]. Compared to the placebo group, a significant reduction in mortality was observed in all subgroups. The second endpoint "reduction of hospitalizations due to cardiac decompensation" was achieved in all subgroups-except NYHA III patients. There were also positive effects on the walking distance in the 6-min walking test and on the quality of life in the KCCQ-OS questionnaire. The approved dosage for treatment of amyloid cardiomyopathy is Tafamidis $61 \mathrm{mg}$, the bioequivalent of $80 \mathrm{mg}$ Tafamidis-meglumine. Serious side effects are not expected under treatment with tafamidis [119]. The study data suggest that starting therapy as early as possible has a positive effect on patient outcome. Hence, treatment should be initiated as early as possibleif all diagnostic and study criteria are met. For patients in advanced stages (NYHA III), the decision to start Tafamidis therapy should be made after careful individual assessment and in consideration of comorbidities, general condition, and inclusion/exclusion criteria of the ATTR-ACT study. No data are available for heart failure NYHA class IV.

Since 2018, two TTR-mRNA interfering drugs, the RNA interference (RNAi) drug patisiran $\left(\right.$ Onpattro $\left.{ }^{\circledR}\right)$ [120] and the antisense oligonucleotide inotersen $\left(\right.$ Tegsedi $\left.^{\circledR}\right)$ [121], have been approved in Europe, both for the treatment of ATTRv polyneuropathy in stages I-II. In both pivotal studies, the primary neurological endpoints were met. Since patients with mixed phenotype were also included, first subgroup analyses for patients with cardiac involvement exist. Patisiran showed a significant reduction of LV wall thickness and NT-proBNP levels in a subgroup of ATTRv patients [122]. However, since in this study, cardiac amyloidosis was confirmed neither bioptically nor by means of CMR-based or scintigraphic methods, these initial results should be interpreted with caution.

Despite slight differences between the study cohorts, it can be assumed that, based on the study results to date, both patisiran and inotersen are equally effective and well tolerated. Since the use of these drugs is currently only possible for neurological manifestation, an interdisciplinary approach is obligatory. Positive effects on the progression of cardiomyopathy should be expected for both drugs on the basis of the previous subgroup analyses. Reliable study data regarding the prognostic relevance in patients with cardiac amyloidosis are not yet available, but two clinical trials were recently initiated to evaluate the safety and efficacy of RNAi drugs for the treatment of amyloid cardiomyopathy (NCT0413671 and NCT04153149).

Furthermore, there is some evidence that the polyphenol epigallocatechin gallate (EGCG) may have a positive effect on the clinical course of both types of cardiac ATTR amyloidosis [123], although peer-reviewed results of the corresponding prospective randomized studies are not yet available. It can be taken as a dietary supplement and a dose of 800-1200 mg EGCG with added vitamin C is recommended. Positive effects have also been described for the 
combination of doxycycline and Urso-falk ${ }^{\circledR}$ [124], which can be used as off-label therapy.

Obviously, addressing cost-effectiveness issues for novel ATTR therapies is not within the scope of this position statement. However, we encourage cost-effectiveness analyses such as performed recently by Kazi et al. [125] for use of Tafamidis (in ATTR cardiomyopathy) -illustrating that a price reduction of more than $90 \%$ is required to make Tafamidis cost-effective at $\$ 100,000$ per quality-adjusted lifeyear (QALE). Considering the growing number of patients with cardiac amyloidosis (in particular with ATTRwt), costeffectiveness issues need to be carefully considered in the future regarding specific, however expensive, drugs addressing amyloid cardiomyopathy.

\section{Specific drug therapies for AA amyloidosis}

Due to the diversity of the various triggering underlying diseases, there is no generally valid treatment strategy for AA amyloidosis. The fundamental and common goal of all therapies is the inhibition of the synthesis of the precursor protein SAA [126]. Depending on the underlying disease, various drugs are used, such as anti-infectives for infectiousinflammatory diseases, immunosuppressants for autoimmune diseases, and chemotherapy for malignant diseases. The target of newer therapies is the precursor protein itself; initial results are available, for example, for the monoclonal anti-IL-6 antibody tocilizumab. There is no specific drug therapy for heart involvement.

\section{Liver transplantation in ATTR amyloidosis}

Transthyretin, the protein underlying ATTR amyloidosis, is produced almost exclusively in the liver. Hence, liver transplantation is a possible treatment option for patients with ATTRv [127]. The genuine liver, which produces the variant form of transthyretin, is replaced by a donor liver that synthesizes the wild-type form of transthyretin. After transplantation, there is no further production of variant transthyretin, and the progression of neurological as well as of cardiac symptoms should be stopped $[128,129]$. The liver of a mutation carrier which is healthy in terms of detoxification and synthesis performance can be passed on to a patient with another liver disease on the transplant waiting list in the sense of a domino liver transplant. However, it should be noted that the organ recipient will also develop ATTRv amyloidosis in the long term.

According to the most recent figures of the worldwide "Familial World Transplant Registry" (http://fapwtr.org), approximately 120 patients with ATTRv receive a liver transplant per year, and by 2017 , a total of 68 ATTRv patients received liver transplants in Germany. This therapeutic option is associated with a 10-year survival rate of
21-85\% depending on the underlying mutation. Independent factors for a favourable outcome are a higher body mass index, an early onset of the disease (age $<50$ years), a short disease duration, and the presence of a Val30Met mutation [128].

In some ATTRv patients, especially those with other than p.Val50Met mutations, further disease progression was observed even after liver transplantation [129]. Mass spectrometric analysis of tissue from affected patients showed a deposition of wild-type transthyretin on existing ATTRv amyloid. Therefore, liver transplantation should be performed at the earliest possible disease stage with a still low level of amyloid deposition. On the other hand, it seems reasonable to treat ATTRv patients with transthyretinstabilizing drugs even after liver transplantation. In patients with advanced cardiac ATTRv amyloidosis, a combined or sequential transplantation of liver and heart can be considered [130]. Of note, a paradoxically accelerated amyloid deposition in the myocardium following a liver transplantation can also occur [131].

Nevertheless, with the approval of new gene-silencing drugs, which eliminate variant transthyretin from the blood [132], analogous to liver transplantation, the indication for liver transplantation must be critically reviewed, especially in patients with other than Val30Met mutations [128]. However, no comparative studies are available to date.

ATTRwt amyloidosis, on the other hand, is based on the deposition of non-mutated wild-type transthyretin, which would be produced in the same way by the donor organ even after a liver transplant. Liver transplantation is therefore not a therapeutic option for this type of amyloidosis.

\section{Device therapy for cardiac amyloidosis}

The clinical decision for or against a device therapy (e.g., pacemaker or ICD) in a patient with cardiac amyloidosis is not easy, since there is a lack of robust evidence-based studies and usually a decision has to be made on a case-bycase basis, carefully considering the individual constellation of findings. On one hand, the type of amyloidosis, the stage of disease, and individual prognosis must be taken into account. In principle, device therapy is only considered if a median life expectancy of at least 1 year is to be expectedwhich should be carefully considered in advanced amyloidosis with cardiac involvement. On the other hand, a comprehensive cardiac evaluation should have been carried out and it should also be taken into account whether special or specific therapies are pending (e.g., chemotherapy for AL amyloidosis).

Common causes of death in patients with cardiac amyloidosis are a (rapidly) progressive heart failure and sudden cardiac death. While some studies have documented a high incidence of ventricular arrhythmias and have even shown 
a prognostic significance for the occurrence of ventricular couplets or nsVTs [133,134], other studies indicate a greater significance of bradycardic arrhythmias (especially higher grade AV blockages or electromechanical decoupling) as triggering factors for decompensation or sudden cardiac death [135]. A causally obvious benefit of pacemaker or ICD therapy (e.g., in the form of a reduction in mortality or SCD risk) has so far not been observed in larger studies $[136,137]$. However, it needs to be considered that in individual cases and smaller studies, convincing evidence of the benefit of ICD therapy in patients with cardiac amyloidosis has been provided [134, 138, 139].

Due to those limited and sometimes contradictory data, the recommendations of the European and American societies have also been divergent in the past. While the ESC guidelines from 2015 only recommended secondary prophylactic ICD implantation in patients with amyloidosis and documented persistent ventricular arrhythmia (strength of recommendation IIa/C) [140], the American guidelines from 2013 also mentioned primary prophylactic indications [141] (including amyloidosis patients with limited LV function (LVEF $<50 \%$ ) and unclear syncope). However, in the American guidelines updated in 2017, these recommendations were withdrawn; instead, only an individual decision is recommended [142]. Recently published recommendations from the Heart Rhythm Society (HRS) suggest ICD implantation in patients with AL amyloidosis in case of nsVTs and an expected survival of $>1$ year ( $\mathrm{IIb} / \mathrm{C})$ - but do not separately address patients with ATTR amyloidosis [143].

Meanwhile, some laboratory parameters (e.g., troponin or NT-proBNP) and imaging parameters (e.g., ECV extent in CMR imaging) exist, which allow better risk stratification with regard to mortality and sudden cardiac death (SCD). In view of the limited data available to date and the anticipated longer life expectancy due to new drug therapy options, the authors of this position statement recommend in addition to the above-mentioned ESC recommendations (a) also a careful decision on a case-by-case basis and (b) a rather generous (primary prophylactic) indication for ICD implantation in patients with an increased mortality risk according to serum or imaging parameters and/or documented nsVTs if the life expectancy is $>1$ year.

For cardiac resynchronization therapy (CRT), there are no reliable data for patients with cardiac amyloidosis. In a few individual cases, the benefits of a CRT have also been shown for these patients, so that, in principle, a CRT can also be

Table 3 Follow-up strategy for cardiac amyloidosis

\begin{tabular}{|c|c|c|}
\hline & AL amyloidosis* & ATTR amyloidosis \\
\hline $\begin{array}{l}\text { During specific drug } \\
\text { therapy (possibly } \\
\text { also "off-label } \\
\text { use") }\end{array}$ & $\begin{array}{l}\text { Every } 3 \text { months (or after every } 2 \text { further } \\
\text { therapy cycles): } \\
\text { NT-proBNP } \\
\text { Troponin T or I } \\
\text { Treatment success with a drop of }>30 \% \text { and } \\
\text { indication of treatment failure if increase } \\
\text { of }>30 \% \text { compared to the respective previous } \\
\text { value } \\
\text { Every } 6 \text { months: } \\
\text { Resting ECG + Holter ECG } \\
\text { Transthoracic echocardiography including strain } \\
\text { measurements } \\
\text { If available: CMR including LGE and T1 map- } \\
\text { ping }\end{array}$ & $\begin{array}{l}\text { Every 3-6 months: } \\
\text { NT-proBNP } \\
\text { Troponin T or I } \\
\text { Assessment of treatment success depends on the respective drug } \\
\text { Every } 12 \text { months: } \\
\text { Resting ECG + Holter ECG } \\
\text { Transthoracic echocardiography including strain measurements } \\
\text { If available: CMR including LGE and T1 mapping }\end{array}$ \\
\hline $\begin{array}{l}\text { After remission or } \\
\text { in stable condition } \\
\text { without specific } \\
\text { therapy }\end{array}$ & $\begin{array}{l}\text { Every } 6 \text { months: } \\
\text { Resting ECG } \\
\text { NT-proBNP } \\
\text { Troponin T or I } \\
\text { Transthoracic echocardiography including strain } \\
\text { measurements } \\
\text { Every } 12 \text { months: } \\
\text { Holter ECG } \\
\text { Additional CMR including LGE and T1 map- } \\
\text { ping in case of suspected disease progression } \\
\text { due to serum biomarkers and/or echocardio- } \\
\text { graphic findings }\end{array}$ & $\begin{array}{l}\text { Every } 6 \text { months: } \\
\text { Resting ECG } \\
\text { NT-proBNP } \\
\text { Troponin T or I } \\
\text { Transthoracic echocardiography including strain measurements } \\
\text { Every } 12 \text { months: } \\
\text { Holter ECG } \\
\text { Every } 12 \text { to } 24 \text { months: } \\
\text { Additional CMR including LGE and T1 mapping in case of suspected } \\
\text { disease progression due to serum biomarkers and/or echocardio- } \\
\text { graphic findings }\end{array}$ \\
\hline
\end{tabular}

\footnotetext{
*In addition to the criteria regarding cardiac disease response, hematologic response criteria should be measured every 3 months

${ }^{a}$ Measuring of NT-proBNP should be performed in patients in a (re)compensated state (and with a time delay from any cortisone administration)

${ }^{\mathrm{b}}$ For Tafamidis, a lack of a further increase in NT-proBNP under therapy has already been interpreted as a therapeutic success, while, for example, under Patisiran, a drop of $>30 \%$ to the respective previous value was described after 9 and 18 months $[88,92]$
} 
considered for amyloidosis patients and the current recommendations for the CRT indication should be applied [144].

\section{Clinical follow-up in cardiac amyloidosis}

In patients with amyloidosis, serial comprehensive follow-up examinations should be performed including the assessment of all involved organs/organ systems and, in AL amyloidosis, of the hematopoietic system. In AL amyloidosis, the hematologic response often precedes organ response and guides the way regarding optimal hematologic treatment.

For the assessment of the clinical course of cardiac amyloidosis and the respective treatment effect, primarily laboratory biomarkers such as NT-proBNP or troponin T (or -I) as well as non-invasive imaging parameters are available $[113,145]$.

Even though (a) the existing data for serum biomarkers such as NT-proBNP or troponin T (or I) are much more comprehensive than for non-invasive imaging parameters (e.g., CMR parameters) [146] and (b) some staging procedures already take into account the level of NT-proBNP or troponin T (or I) levels [37], a diagnostic "superiority" of these serum parameters in direct comparison to new imaging parameters has not yet been proven. On the contrary, initial CMR results indicate that a more precise risk stratification, for example, with the T1 mapping procedure and the determination of the ECV value, is possible [70]. Simply measuring the LV wall thickness or the LVEF, on the other hand, is insufficient or only of minor importance with regard to the assessment of the success of treatment and to risk stratification of cardiac amyloidosis. For this reason, we currently recommend the relatively comprehensive follow-up mentioned in Table 3 for patients with cardiac amyloidosis, depending on the respective constellation. Further research on this important aspect is urgently indicated.

\section{Summary}

Amyloidosis can affect both sexes and individuals of any age. As a multi-organ disease presenting with a variety of rather unspecific symptoms of different severity, the diagnosis of amyloidosis often is delayed, and the number of unreported cases is probably high. Therefore, interdisciplinary cooperation between neurology, hematology, gastroenterology, pathology, and cardiology (and other specialist groups as needed) is an indispensable prerequisite for successful care of the individual patient.

In principle, amyloid can infiltrate all structures of the heart and affect not only the ventricular and atrial walls but also the conduction system, the heart valves, and the coronaries. Therefore, the clinical spectrum of cardiovascular involvement is wide and ranges from asymptomatic courses of dizziness and syncope to the development of restrictive cardiomyopathy and progressive terminal heart failure.

However, the different types of amyloid show different organ tropism typical for the respective disease. Systemic forms of amyloidosis, which affect the heart, are mainly the light chain (AL) and ATTR amyloidoses, resulting from the deposition of misfolded transthyretin (either as the wild-type [ATTRwt] or mutated [ATTRv] form).

In addition to specific cardiac biomarkers, modern noninvasive imaging techniques such as CMR or ${ }^{99 \mathrm{~m}} \mathrm{Tc}$-phosphate scintigraphy are available today, which complement conventional echocardiography and allow not only for the diagnosis of cardiac amyloidosis but also an exact determination of the degree of its manifestation. As an invasive diagnostic procedure, endomyocardial biopsy continues to play a central role in the histopathological confirmation and subtyping of cardiac amyloidosis.

The main objective of the diagnostic path outlined in this position statement is to detect the presence of cardiac amyloidosis as reliably and early as possible, to precisely characterize the extent of cardiac amyloidosis, and subsequently to enable targeted treatment (including the possibility of monitoring the success of therapy) - in consideration of local experience and expertise.

Finally, the targeted treatment of cardiac amyloidosis is increasingly in the focus of clinical trials, and in addition to a label extension for already available substances, the approval of additional newer drugs is expected in the near future.

Acknowledgements We would like to thank Stefan Knop from the Department of Medicine II, Würzburg University Hospital, for his support in reviewing hematological aspects of this manuscript.

Funding Open Access funding enabled and organized by Projekt DEAL.

\section{Compliance with ethical standards}

Conflict of interest Ali Yilmaz: speaker and consultant activities for Alnylam, Pfizer Pharma GmbH and Akcea Therapeutics; research cooperation with Philips. Johann Bauersachs: speaker and consultant activities for Pfizer Pharma GmbH. Frank Bengel: speaker and consultant activities for Siemens Healthineers, GE Healthcare, Alnylam and Pfizer Pharma GmbH. Ronny Büchel: speaker honoraria from Pfizer Pharma GmbH and GE Healthcare. Ingrid Kindermann: speaker honoraria from Akcea Therapeutics, Alnylam and Pfizer Pharma GmbH. Karin Klingel: speaker honoraria from Akcea Therapeutics, Alnylam and Pfizer Pharma GmbH. Fabian Knebel: speaker and consultant activities for Akcea Therapeutics, Alnylam and Pfizer Pharma $\mathrm{GmbH}$. Benjamin Meder and Fabian aus dem Siepen: speaker honoraria from Akcea Therapeutics, Pfizer Pharma GmbH and Alnylam as well as travel grants from Pfizer Pharma GmbH. Caroline Morbach: research cooperation with the University of Würzburg and Tomtec Im- 
aging Systems funded by a research grant from the Bavarian Ministry of Economic Affairs, Regional Development and Energy, Germany; speakers honorarium and travel grants from Amgen, Tomtec, Orion Pharma, and Alnylam; participation in Advisory and Patient Eligibility Boards sponsored by Akcea, Alnylam, Pfizer, and EBR Systems; principal investigator in trials sponsored by Alnylam and AstraZeneca. Eike Nagel: speaker honoraria from Bayer AG and Neosoft; research cooperation with Bayer AG. Eric Schulze-Bahr: None. Norbert Frey: None.

Open Access This article is licensed under a Creative Commons Attribution 4.0 International License, which permits use, sharing, adaptation, distribution and reproduction in any medium or format, as long as you give appropriate credit to the original author(s) and the source, provide a link to the Creative Commons licence, and indicate if changes were made. The images or other third party material in this article are included in the article's Creative Commons licence, unless indicated otherwise in a credit line to the material. If material is not included in the article's Creative Commons licence and your intended use is not permitted by statutory regulation or exceeds the permitted use, you will need to obtain permission directly from the copyright holder. To view a copy of this licence, visit http://creativecommons.org/licenses/by/4.0/.

\section{References}

1. Vaxman I, Gertz M (2019) Recent advances in the diagnosis, risk stratification, and management of systemic light-chain amyloidosis. Acta Haematol 141(2):93-106

2. Ihne S, Morbach C, Obici L, Palladini G, Störk S (2019) Amyloidosis in heart failure. Curr Heart Fail Rep 16(6):285-303

3. Palladini G, Russo P, Bosoni T, Verga L, Sarais G, Lavatelli F, Nuvolone M, Obici L, Casarini S, Donadei S, Albertini R, Righetti G, Marini M, Graziani MS, Melzi D'Eril GV, Moratti R, Merlini G (2009) Identification of amyloidogenic light chains requires the combination of serum-free light chain assay with immunofixation of serum and urine. Clin Chem 55(3):499-504. https://doi.org/10.1373/clinchem.2008.117143

4. Ablasser K, Verheyen N, Glantschnig T, Agnetti G, Rainer PP (2019) Unfolding cardiac amyloidosis-from pathophysiology to cure. Curr Med Chem 26(16):2865-2878. https://doi. org/10.2174/0929867325666180104153338

5. Hammarstrom P, Jiang X, Hurshman AR, Powers ET, Kelly JW (2002) Sequence-dependent denaturation energetics: a major determinant in amyloid disease diversity. Proc Natl Acad Sci USA 99(Suppl 4):16427-16432

6. Merlini G, Bellotti V (2003) Molecular mechanisms of amyloidosis. N Engl J Med 349(6):583-596

7. Bourgault S, Choi S, Buxbaum JN, Kelly JW, Price JL, Reixach N (2011) Mechanisms of transthyretin cardiomyocyte toxicity inhibition by resveratrol analogs. Biochem Biophys Res Commun 410(4):707-713

8. Rocken C, Shakespeare A (2002) Pathology, diagnosis and pathogenesis of AA amyloidosis. Virchows Arch 440(2):111-122

9. Gilstrap LG, Dominici F, Wang Y, E-Sady MS, Singh A, Di Carli MF, Falk RH, Dorbala S (2019) Epidemiology of cardiac amyloidosis-associated heart failure hospitalizations among fee-for-service Medicare beneficiaries in the United States. Circ Heart Fail 12(6):e005407. https://doi.org/10.1161/CIRCH EARTFAILURE.118.005407

10. Quock TP, Yan T, Chang E, Guthrie S, Broder MS (2018) Epidemiology of AL amyloidosis: a realworld study using US claims data. Blood Adv 2(10):1046-1053
11. Kyle RA, Linos A, Beard CM, Linke RP, Gertz MA, O'Fallon WM, Kurland LT (1992) Incidence and natural history of primary systemic amyloidosis in Olmsted county, Minnesota, 1950 through 1989. Blood 79(7):1817-1822

12. Madan S, Kumar SK, Dispenzieri A, Lacy MQ, Hayman SR, Buadi FK, Dingli D, Rajkumar SV, Hogan WJ, Leung N, Grogan M, Gertz MA (2012) High-dose melphalan and peripheral blood stem cell transplantation for light-chain amyloidosis with cardiac involvement. Blood 119(5):1117-1122

13. Cornwell GG III, Murdoch WL, Kyle RA, Westermark P, Pitkanen P (1983) Frequency and distribution of senile cardiovascular amyloid A clinicopathologic correlation. Am J Med 75(4):618-623

14. Gonzalez-Lopez E, Gallego-Delgado M, Guzzo-Merello G, de Haro-Del Moral FJ, Cobo-Marcos M, Robles C, Bornstein B, Salas C, Lara-Pezzi E, Alonso-Pulpon L, Garcia-Pavia P (2015) Wild-type transthyretin amyloidosis as a cause of heart failure with preserved ejection fraction. Eur Heart J 36(38):2585-2594

15. Mohammed SF, Mirzoyev SA, Edwards WD, Dogan A, Grogan DR, Dunlay SM, Roger VL, Gertz MA, Dispenzieri A, Zeldenrust SR, Redfield MM (2014) Left ventricular amyloid deposition in patients with heart failure and preserved ejection fraction. JACC Heart Fail 2(2):113-122

16. Koivuniemi R, Paimela L, Suomalainen R, Tornroth T, LeirisaloRepo M (2008) Amyloidosis is frequently undetected in patients with rheumatoid arthritis. Amyloid 15(4):262-268

17. Rapezzi C, Lorenzini M, Longhi S, Milandri A, Gagliardi C, Bartolomei I, Salvi F, Maurer MS (2015) Cardiac amyloidosis: the great pretender. Heart Fail Rev 20(2):117-124

18. Desport E, Bridoux F, Sirac C, Delbes S, Bender S, Fernandez B, Quellard N, Lacombe C, Goujon JM, Lavergne D, Abraham J, Touchard G, Fermand JP, Jaccard A (2012) Al amyloidosis. Orphanet J Rare Dis 7:54

19. Feng D, Edwards WD, Oh JK, Chandrasekaran K, Grogan M, Martinez MW, Syed IS, Hughes DA, Lust JA, Jaffe AS, Gertz MA, Klarich KW (2007) Intracardiac thrombosis and embolism in patients with cardiac amyloidosis. Circulation 116(21):2420-2426

20. Tsai SB, Seldin DC, Wu H, O'Hara C, Ruberg FL, Sanchorawala V (2011) Myocardial infarction with "clean coronaries" caused by amyloid light-chain AL amyloidosis: a case report and literature review. Amyloid 18(3):160-164

21. Dorbala S, Vangala D, Bruyere J Jr, Quarta C, Kruger J, Padera R, Foster C, Hanley M, Di Carli MF, Falk R (2014) Coronary microvascular dysfunction is related to abnormalities in myocardial structure and function in cardiac amyloidosis. JACC Heart Fail 2(4):358-367

22. Lousada I, Comenzo RL, Landau H, Guthrie S, Merlini G (2015) Light chain amyloidosis: patient experience survey from the Amyloidosis Research Consortium. Adv Ther 32(10):920-928

23. Kumar S, Dispenzieri A, Lacy MQ, Hayman SR, Buadi FK, Colby C, Laumann K, Zeldenrust SR, Leung N, Dingli D, Greipp PR, Lust JA, Russell SJ, Kyle RA, Rajkumar SV, Gertz MA (2012) Revised prognostic staging system for light chain amyloidosis incorporating cardiac biomarkers and serum free light chain measurements. J Clin Oncol 30(9):989-995

24. Dittrich T, Benner A, Kimmich C, Aus dem Siegen F, Veelken K, Kristen AV, Bochtler T, Katus HA, Müller-Tidow C, Hegenbart U, Schönland SO (2019) Performance analysis of AL amyloidosis cardiac biomarker staging systems with special focus on renal failure and atrial arrhythmia. Haematologica 104(7):1451-1459

25. Rapezzi C, Quarta CC, Riva L, Longhi S, Gallelli I, Lorenzini M, Ciliberti P, Biagini E, Salvi F, Branzi A (2010) Transthyretin-related amyloidoses and the heart: a clinical overview. Nat Rev Cardiol 7(7):398-408 
26. Rapezzi C, Merlini G, Quarta CC, Riva L, Longhi S, Leone O, Salvi F, Ciliberti P, Pastorelli F, Biagini E, Coccolo F, Cooke RM, Bacchi-Reggiani L, Sangiorgi D, Ferlini A, Cavo M, Zamagni E, Fonte ML, Palladini G, Salinaro F, Musca F, Obici L, Branzi A, Perlini S (2009) Systemic cardiac amyloidoses: disease profiles and clinical courses of the 3 main types. Circulation 120(13):1203-1212

27. Maurer MS, Elliott P, Comenzo R, Semigran M, Rapezzi C (2017) Addressing common questions encountered in the diagnosis and management of cardiac amyloidosis. Circulation 135(14): 1357-1377

28. Nativi-Nicolau J, Maurer MS (2018) Amyloidosis cardiomyopathy: update in the diagnosis and treatment of the most common types. Curr Opin Cardiol 33(5):571-579

29. Donnellan E, Wazni OM, Saliba WI, Hanna M, Kanj M, Patel DR, Wilner B, Kochar A, Jaber WA (2020) Prevalence, incidence, and impact on mortality of conduction system disease in transthyretin cardiac amyloidosis. Am J Cardiol 128:140-146

30. Ruberg FL, Grogan M, Hanna M, Kelly JW, Maurer MS (2019) Transthyretin amyloid cardiomyopathy: JACC state-of-the-art review. J Am Coll Cardiol 73(22):2872-2891

31. Cavalcante JL, Rijal S, Abdelkarim I, Althouse AD, Sharbaugh MS, Fridman Y, Soman P, Forman DE, Schindler JT, Gleason TG, Lee JS, Schelbert EB (2017) Cardiac amyloidosis is prevalent in older patients with aortic stenosis and carries worse prognosis. J Cardiovasc Magn Reson 19(1):98

32. Nitsche C, Scully PR, Patel KP, Kammerlander A, Koschutnik M, Dona C, Wollenweber T, Ahmed N, Thornton GD, Kelion A, Sabharwal N, Newton JD, Ozkor M, Kennon S, Mullen M, Lloyd G, Fontana M, Hawkins P, Pugliese F, Menezes L, Moon JC, Mascherbauer J, Treibel TA (2021) Prevalence and outcomes of concomitant aortic stenosis and cardiac amyloidosis. J Am Coll Cardiol 77:128-139. https://doi.org/10.1016/j. jacc.2020.11.006

33. Castano A, Narotsky DL, Hamid N, Khalique OK, Morgenstern R, DeLuca A, Rubin J, Chiuzan C, Nazif T, Vahl T, George I, Kodali S, Leon MB, Hahn R, Bokhari S, Maurer MS (2017) Unveiling transthyretin cardiac amyloidosis and its predictors among elderly patients with severe aortic stenosis undergoing transcatheter aortic valve replacement. Eur Heart J 38(38):2879-2887

34. Treibel TA, Fontana M, Gilbertson JA, Castelletti S, White SK, Scully PR, Roberts N, Hutt DF, Rowczenio DM, Whelan CJ, Ashworth MA, Gillmore JD, Hawkins PN, Moon JC (2016) Occult transthyretin cardiac amyloid in severe calcific aortic stenosis: prevalence and prognosis in patients undergoing surgical aortic valve replacement. Circ Cardiovasc Imaging 9(8):e005066. https://doi.org/10.1161/CIRCIMAGIN G.116.005066

35. Maurer MS, Hanna M, Grogan M, Dispenzieri A, Witteles R, Drachman B, Judge DP, Lenihan DJ, Gottlieb SS, Shah SJ, Steidley DE, Ventura H, Murali S, Silver MA, Jacoby D, Fedson S, Hummel SL, Kristen AV, Damy T, Plante-Bordeneuve V, Coelho T, Mundayat R, Suhr OB, Waddington CM, Rapezzi C (2016) Genotype and phenotype of transthyretin cardiac amyloidosis: THAOS (Transthyretin Amyloid Outcome Survey). J Am Coll Cardiol 68(2):161-172

36. Ruberg FL, Maurer MS, Judge DP, Zeldenrust S, Skinner M, Kim AY, Falk RH, Cheung KN, Patel AR, Pano A, Packman J, Grogan DR (2012) Prospective evaluation of the morbidity and mortality of wild-type and V122I mutant transthyretin amyloid cardiomyopathy: the Transthyretin Amyloidosis Cardiac Study (TRACS). Am Heart J 164(2):222-228

37. Grogan M, Scott CG, Kyle RA, Zeldenrust SR, Gertz MA, Lin G, Klarich KW, Miller WL, Maleszewski JJ, Dispenzieri A (2016) Natural history of wild-type transthyretin cardiac amyloidosis and risk stratification using a novel staging system. J Am Coll Cardiol 68(10):1014-1020

38. Gillmore JD, Damy T, Fontana M, Hutchinson M, Lachman HJ, Martinez-Naharro A, Quarta CC, Rezk T, Whelan CJ, GonzalezLopez E, Lane T, Gilberson JA, Rowczenio D, Petrie A, Hawkins PN (2018) A new staging system for cardiac transthyretin amyloidosis. Eur Heart J 39(30):2799-2806

39. Connors LH, Sam F, Skinner M, Salinaro F, Sun F, Ruberg FL, Berk JL, Seldin DC (2016) Heart failure resulting from agerelated cardiac amyloid disease associated with wild-type transthyretin: a prospective, observational cohort study. Circulation 133(3):282-290

40. Janssen S, van Rijswijk MH, Meijer S, Ruinen L, Van der Hem GK (1986) Systemic amyloidosis: a clinical survey of 144 cases. Neth J Med 29(11):376-385

41. Lachmann HJ, Goodman HJ, Gilbertson JA, Gallimore JR, Sabin CA, Gillmore JD, Hawkins PN (2007) Natural history and outcome in systemic AA amyloidosis. N Engl J Med 356(23):2361-2371

42. Tanaka F, Migita K, Honda S, Fukuda T, Mine M, Nakamura T, Yamasaki S, Ida H, Kawakami A, Origuchi T, Eguchi K (2003) Clinical outcome and survival of secondary (AA) amyloidosis. Clin Exp Rheumatol 21(3):343-346

43. Bergesio F, Ciciani AM, Manganaro M, Palladini G, Santostefano M, Brugnano R, Di Palma AM, Gallo M, Rosati A, Tosi PL, Salvadori M (2008) Renal involvement in systemic amyloidosis: an Italian collaborative study on survival and renal outcome. Nephrol Dial Transplant 23(3):941-951

44. Gillmore JD, Damy T, Fontana M, Hutchinson M, Lachmann HJ, Martinez-Naharro A, Quarta CC, Rezk T, Whelan CJ, GonzalezLopez E, Lane T, Gilbertson JA, Rowczenio D, Petrie A, Hawkins PN (2018) A new staging system for cardiac transthyretin amyloidosis. Eur Heart J 39(30):2799-2806

45. Hanson JLS, Arvanitis M, Koch CM, Berk JL, Ruberg FL, Prokaeva T, Connors LH (2018) Use of serum transthyretin as a prognostic indicator and predictor of outcome in cardiac amyloid disease associated with wild-type transthyretin. Circ Heart Fail 11(2): 0004000

46. Gertz MA (2018) Immunoglobulin light chain amyloidosis: 2018 update on diagnosis, prognosis, and treatment. Am J Hematol 93(9):1169-1180

47. Sperry BW, Vranian MN, Hachamovitch R, Joshi H, McCarthy M, Ikram A, Hanna M (2016) Are classic predictors of voltage valid in cardiac amyloidosis? A contemporary analysis of electrocardiographic findings. Int J Cardiol 214:477-481

48. Namdar M, Steffel J, Jetzer S, Schmied C, Hurlimann D, Camici GG, Bayrak F, Ricciardi D, Rao JY, de Asmundis C, Chierchia GB, Sarkozy A, Luscher TF, Jenni R, Duru F, Brugada P (2012) Value of electrocardiogram in the differentiation of hypertensive heart disease, hypertrophic cardiomyopathy, aortic stenosis, amyloidosis, and Fabry disease. Am J Cardiol 109(4):587-593

49. van den Berg MP, Mulder BA, Klaassen SHC, Maass AH, Van Veldhuisen DJ, van der Meer P, Nienhuis HLA, Hazenberg BPC, Rienstra M (2019) Heart failure with preserved ejection fraction, atrial fibrillation, and the role of senile amyloidosis. Eur Heart $\mathbf{J}$ 40(16):1287-1293. https://doi.org/10.1093/eurheartj/ehz057

50. Brignole M, Moya A, de Lange FJ, Deharo JC, Elliott PM, Fanciulli A, Fedorowski A, Furlan R, Kenny RA, Martin A, Probst V, Reed MJ, Rice CP, Sutton R, Ungar A, van Dijk JG (2018) 2018 ESC Guidelines for the diagnosis and management of syncope. Eur Heart J 39(21):1883-1948

51. Navarro JF, Rivera M, Ortuno J (1992) Cardiac tamponade as presentation of systemic amyloidosis. Int $\mathrm{J}$ Cardiol 36(1):107-108

52. Lang RM, Badano LP, Mor-Avi V, Afilalo J, Armstrong A, Ernande L, Flachskampf FA, Foster E, Goldstein SA, Kuznetsova 
T, Lancellotti P, Muraru D, Picard MH, Rietzschel ER, Rudski L, Spencer KT, Tsang W, Voigt JU (2015) Recommendations for cardiac chamber quantification by echocardiography in adults: an update from the American Society of Echocardiography and the European Association of Cardiovascular Imaging. Eur Heart J Cardiovasc Imaging 16(3):233-270

53. Ponikowski P, Voors AA, Anker SD, Bueno H, Cleland JGF, Coats AJS, Falk V, Gonzalez-Juanatey JR, Harjola VP, Jankowska EA, Jessup M, Linde C, Nihoyannopoulos P, Parissis JT, Pieske B, Riley JP, Rosano GMC, Ruilope LM, Ruschitzka F, Rutten FH, van der Meer P (2016) 2016 ESC Guidelines for the diagnosis and treatment of acute and chronic heart failure: The Task Force for the diagnosis and treatment of acute and chronic heart failure of the European Society of Cardiology (ESC). Developed with the special contribution of the Heart Failure Association (HFA) of the ESC. Eur Heart J 37(27):2129-2200

54. Phelan D, Collier P, Thavendiranathan P, Popovic ZB, Hanna M, Plana JC, Marwick TH, Thomas JD (2012) Relative apical sparing of longitudinal strain using two-dimensional speckle-tracking echocardiography is both sensitive and specific for the diagnosis of cardiac amyloidosis. Heart 98(19):1442-1448

55. Ternacle J, Bodez D, Guellich A, Audureau E, Rappeneau S, Lim P, Radu C, Guendouz S, Couetil JP, Benhaiem N, Hittinger L, Dubois-Rande JL, Plante-Bordeneuve V, Mohty D, Deux JF, Damy T (2016) Causes and consequences of longitudinal LV dysfunction assessed by 2D strain echocardiography in cardiac amyloidosis. JACC Cardiovasc Imaging 9(2):126-138

56. Pagourelias ED, Mirea O, Duchenne J, Van CJ, Delforge M, Bogaert J, Kuznetsova T, Voigt JU (2017) Echo parameters for differential diagnosis in cardiac amyloidosis: a head-to-head comparison of deformation and nondeformation parameters. Circ Cardiovasc Imaging 10(3):e005588

57. Senior R, Becher H, Monaghan M, Agati L, Zamorano J, Vanoverschelde JL, Nihoyannopoulos P, Edvardsen T, Lancellotti P (2017) Clinical practice of contrast echocardiography: recommendation by the European Association of Cardiovascular Imaging (EACVI) 2017. Eur Heart J Cardiovasc Imaging 18(11):1205-1205af

58. Claus P, Omar AMS, Pedrizzetti G, Sengupta PP, Nagel E (2015) Tissue tracking technology for assessing cardiac mechanics: principles, normal values, and clinical applications. JACC Cardiovasc Imaging 8(12):1444-1460

59. Maceira AM, Prasad SK, Hawkins PN, Roughton M, Pennell DJ (2008) Cardiovascular magnetic resonance and prognosis in cardiac amyloidosis. J Cardiovasc Magn Reson 10(1):54

60. Syed IS, Glockner JF, Feng D, Araoz PA, Martinez MW, Edwards WD, Gertz MA, Dispenzieri A, Oh JK, Bellavia D, Tajik AJ, Grogan M (2010) Role of cardiac magnetic resonance imaging in the detection of cardiac amyloidosis. JACC Cardiovasc Imaging 3(2):155-164

61. Vogelsberg H, Mahrholdt H, Deluigi CC, Yilmaz A, Kispert EM, Greulich S, Klingel K, Kandolf R, Sechtem U (2008) Cardiovascular magnetic resonance in clinically suspected cardiac amyloidosis: noninvasive imaging compared to endomyocardial biopsy. J Am Coll Cardiol 51(10):1022-1030

62. White JA, Kim HW, Shah D, Fine N, Kim KY, Wendell DC, Al-Jaroudi W, Parker M, Patel M, Gwadry-Sridhar F, Judd RM, Kim RJ (2014) CMR imaging with rapid visual T1 assessment predicts mortality in patients suspected of cardiac amyloidosis. JACC Cardiovasc Imaging 7(2):143-156

63. Boynton SJ, Geske JB, Dispenzieri A, Syed IS, Hanson TJ, Grogan M, Araoz PA (2016) LGE provides incremental prognostic information over serum biomarkers in AL cardiac amyloidosis. JACC Cardiovasc Imaging 9(6):680-686

64. Fontana M, Pica S, Reant P, Abdel-Gadir A, Treibel TA, Banypersad SM, Maestrini V, Barcella W, Rosmini S, Bulluck H,
Sayed RH, Patel K, Mamhood S, Bucciarelli-Ducci C, Whelan CJ, Herrey AS, Lachmann HJ, Wechalekar AD, Manisty CH, Schelbert EB, Kellman P, Gillmore JD, Hawkins PN, Moon JC (2015) Prognostic value of late gadolinium enhancement cardiovascular magnetic resonance in cardiac amyloidosis. Circulation 132(16):1570-1579

65. Puntmann VO, Peker E, Chandrashekhar Y, Nagel E (2016) T1 mapping in characterizing myocardial disease: a comprehensive review. Circ Res 119(2):277-299

66. Fontana M, Banypersad SM, Treibel TA, Maestrini V, Sado DM, White SK, Pica S, Castelletti S, Piechnik SK, Robson MD, Gilbertson JA, Rowczenio D, Hutt DF, Lachmann HJ, Wechalekar AD, Whelan CJ, Gillmore JD, Hawkins PN, Moon JC (2014) Native T1 mapping in transthyretin amyloidosis. JACC Cardiovasc Imaging 7(2):157-165

67. Hinojar R, Varma N, Child N, Goodman B, Jabbour A, Yu CY, Gebker R, Doltra A, Kelle S, Khan S, Rogers T, Arroyo UE, Cummins C, Carr-White G, Nagel E, Puntmann VO (2015) T1 mapping in discrimination of hypertrophic phenotypes: hypertensive heart disease and hypertrophic cardiomyopathy: findings from the International T1 Multicenter Cardiovascular Magnetic Resonance Study. Circ Cardiovasc Imaging 8(12):e003285

68. Karamitsos TD, Piechnik SK, Banypersad SM, Fontana M, Ntusi NB, Ferreira VM, Whelan CJ, Myerson SG, Robson MD, Hawkins PN, Neubauer S, Moon JC (2013) Noncontrast T1 mapping for the diagnosis of cardiac amyloidosis. JACC Cardiovasc Imaging 6(4):488-497

69. Messroghli DR, Moon JC, Ferreira VM, Grosse-Wortmann L, He T, Kellman P, Mascherbauer J, Nezafat R, Salerno M, Schelbert EB, Taylor AJ, Thompson R, Ugander M, van Heeswijk RB, Friedrich MG (2017) Clinical recommendations for cardiovascular magnetic resonance mapping of $\mathrm{T} 1, \mathrm{~T} 2, \mathrm{~T} 2 *$ and extracellular volume: a consensus statement by the Society for Cardiovascular Magnetic Resonance (SCMR) endorsed by the European Association for Cardiovascular Imaging (EACVI). J Cardiovasc Magn Reson 19(1):75

70. Martinez-Naharro A, Kotecha T, Norrington K, Boldrini M, Rezk T, Quarta C, Treibel TA, Whelan CJ, Knight DS, Kellman P, Ruberg FL, Gillmore JD, Moon JC, Hawkins PN, Fontana M (2018) Native T1 and extracellular volume in transthyretin amyloidosis. JACC Cardiovasc Imaging 12(5):810-819

71. Yilmaz A (2018) The, "native T1 versus extracellular volume fraction paradox" in cardiac amyloidosis: answer to the milliondollar question? JACC Cardiovasc Imaging 12(5):820-822

72. Shintani Y, Okada A, Morita Y, Hamatani Y, Amano M, Takahama H, Amaki M, Hasegawa T, Ohta-Ogo K, Kanzaki H, Ishibasi-Ueda H, Yasuda S, Shimazaki C, Yoshinaga T, Yazaki M, Sekijima Y, Izumi C (2019) Monitoring treatment response to tafamidis by serial native $\mathrm{T} 1$ and extracellular volume in transthyretin amyloid cardiomyopathy. ESC Heart Fail 6(1):232-236

73. Florian A, Bietenbeck M, Chatzantonis G, Hüsing-Kabar A, Schmidt H, Yilmaz A (2020) Regression of cardiac amyloid load documented by cardiovascular magnetic resonance in a patient with hereditary amyloidosis. Clin Res Cardiol 109(7):949-956

74. Nicod P, Lewis SE, Corbett JC, Buja LM, Henderson G, Raskin P, Rude RE, Willerson JT (1982) Increased incidence and clinical correlation of persistently abnormal technetium pyrophosphate myocardial scintigrams following acute myocardial infarction in patients with diabetes mellitus. Am Heart J 103(5):822-829

75. Wizenberg TA, Muz J, Sohn YH, Samlowski W, Weissler AM (1982) Value of positive myocardial technetium-99m-pyrophosphate scintigraphy in the noninvasive diagnosis of cardiac amyloidosis. Am Heart J 103(4 Pt 1):468-473

76. Bokhari S, Castano A, Pozniakoff T, Deslisle S, Latif F, Maurer MS (2013) (99m)Tc-pyrophosphate scintigraphy for differentiating light-chain cardiac amyloidosis from the 
transthyretin-related familial and senile cardiac amyloidoses. Circ Cardiovasc Imaging 6(2):195-201

77. Glaudemans AW, van Rheenen RW, van den Berg MP, Noordzij W, Koole M, Blokzijl H, Dierckx RA, Slart RH, Hazenberg BP (2014) Bone scintigraphy with (99m)technetium-hydroxymethylene diphosphonate allows early diagnosis of cardiac involvement in patients with transthyretin-derived systemic amyloidosis. Amyloid 21(1):35-44

78. Perugini E, Guidalotti PL, Salvi F, Cooke RM, Pettinato C, Riva L, Leone O, Farsad M, Ciliberti P, Bacchi-Reggiani L, Fallani F, Branzi A, Rapezzi C (2005) Noninvasive etiologic diagnosis of cardiac amyloidosis using 99mTc-3,3-diphosphono-1,2-propanodicarboxylic acid scintigraphy. J Am Coll Cardiol 46(6):1076-1084

79. Einstein AJ, Shuryak I, Castaño A, Mintz A, Maurer MS, Bokhari S (2020) Estimating cancer risk from ${ }^{99 \mathrm{~m}}$ Tc pyrophosphate imaging for transthyretin cardiac amyloidosis. J Nucl Cardiol 27(1):215-224

80. Singh V, Falk R, Di Carli MF, Kijewski M, Rapezzi C, Dorbala $S$ (2019) State-of-the-art radionuclide imaging in cardiac transthyretin amyloidosis. J Nucl Cardiol 26(1):158-173

81. Bokhari S, Castaño A, Pozniakoff T, Deslisle S, Latif F, Maurer MS (2013) (99m)Tc-pyrophosphate scintigraphy for differentiating light-chain cardiac amyloidosis from the transthyretinrelated familial and senile cardiac amyloidoses. Circ Cardiovasc Imaging 6(2):195-201

82. Rapezzi C, Quarta CC, Guidalotti PL, Pettinato C, Fanti S, Leone O, Ferlini A, Longhi S, Lorenzini M, Bacchi Reggiani L, Gagliardi C, Gallo P, Villani C, Salvi F (2011) Role of $(99 \mathrm{~m}) \mathrm{Tc}-\mathrm{DPD}$ scintigraphy in diagnosis and prognosis of hereditary transthyretin-related cardiac amyloidosis. JACC Cardiovasc imaging 4(6):659-670

83. Rapezzi C, Quarta CC, Guidalotti PL, Longhi S, Pettinato C, Leone O, Ferlini A, Salvi F, Gallo P, Gagliardi C, Branzi A (2011) Usefulness and limitations of 99mTc-3,3-diphosphono1,2-propanodicarboxylic acid scintigraphy in the aetiological diagnosis of amyloidotic cardiomyopathy. Eur J Nucl Med Mol Imaging 38(3):470-478

84. Galat A, Rosso J, Guellich A, Van Der Gucht A, Rappeneau S, Bodez D, Guendouz S, Tissot C-M, Hittinger L, DuboisRandé J-L, Plante-Bordeneuve V, Itti E, Meignan M, Damy T (2015) Usefulness of (99m)Tc-HMDP scintigraphy for the etiologic diagnosis and prognosis of cardiac amyloidosis. Amyloid 22(4):210-220

85. Treglia G, Glaudemans AWJM, Bertagna F, Hazenberg BPC, Erba PA, Giubbini R, Ceriani L, Prior JO, Giovanella L, Slart RHJA (2018) Diagnostic accuracy of bone scintigraphy in the assessment of cardiac transthyretin-related amyloidosis: a bivariate meta-analysis. Eur J Nucl Med Mol Imaging 45(11):1945-1955

86. Gillmore JA, Maurer MS, Falk RH, Merlini G, Damy T, Dispenzieri A, Wechalekar AD, Berk JL, Quarta C, Grogan M, Lachmann HJ, Bokhari S, Castano A, Dorbala S, Johnson GB, Glaudemans AWJM, Rezk T, Fontana M, Palladini G, Milani P, Guidalotti PL, Flatman K, Lane T, Vonberg FW, Whelan CJ, Moon JC, Ruberg FL, Miller EJ, Hutt DF, Hazenberg BP, Rapezzi C, Hawkins PN (2016) Nonbiopsy diagnosis of cardiac transthyretin amyloidosis. Circulation 133(24):2404-2412

87. Jurcut R, Onciul S, Adam R, Stan C, Coriu D, Rapezzi C, Popescu BA (2020) Multimodality imaging in cardiac amyloidosis: a primer for cardiologists. Eur Heart J Cardiovasc Imaging 21(8):833-844

88. Dorbala S, Ando Y, Bokhari S, Dispenzieri A, Falk RH, Ferrari VA, Fontana M, Gheysens O, Gillmore JD, Glaudemans AWJM, Hanna MA, Hazenberg BPC, Kristen AV, Kwong RY, Maurer MS, Merlkini G, Miller EJ, Moon JC, Murthy VL, Quarta CC,
Rapezzi C, Ruberg FL, Shah SJ, Slart RHJA, Verberne HJ, Bourque JM (2019) ASNC/AHA/ASE/EANM/HFSA/ISA/SCMR/ SNMMI Expert Consensus Recommendations for multimodality imaging in cardiac amyloidosis: Part 1 of 2-evidence base and standardized methods of imaging. J Card Fail 25(11):e1-e39. https://doi.org/10.1016/j.cardfail.2019.08.001

89. Kittleson MM, Maurer MS, Ambardekar AV, Bullock-Palmer RP, Chang PP, Eisen HJ, Nair AP, Nativi-Nicolau J, Ruberg FL (2020) American Heart Association Heart Failure and Transplantation Committee of the Council on Clinical Cardiology. Cardiac amyloidosis: evolving diagnosis and management: a scientific statement from the American Heart Association. Circulation 142(1):e7-e22. https://doi.org/10.1161/CIR.0000000000000792

90. Layoun ME, Desmarais J, Heitner SB, Masri A (2020) Hot hearts on bone scintigraphy are not all amyloidosis: hydroxychloroquine-induced restrictive cardiomyopathy. Eur Heart J 41(25): 2414

91. Pilebro B, Suhr OB, Näslund U, Westermark P, Lindqvist $P$, Sundström T (2016) (99m)Tc-DPD uptake reflects amyloid fibril composition in hereditary transthyretin amyloidosis. Ups J Med Sci 121(1):17-24

92. Ross JC, Hutt DF, Burniston M, Page J, Steeden JA, Gillmore JD, Wechalekar AD, Hawkins PN, Fontana M (2018) Quantitation of $(99 \mathrm{~m}) \mathrm{Tc}-\mathrm{DPD}$ uptake in patients with transthyretin-related cardiac amyloidosis. Amyloid 25(3):203-210

93. Kim YJ, Ha S, Kim YI (2018) Cardiac amyloidosis imaging with amyloid positron emission tomography: A systematic review and meta-analysis. J Nucl Cardiol 27(1):123-132

94. Baratto L, Park SY, Hatami N, Gulaka P, Vasanawala S, Yohannan TK, Herfkens R, Witteles R, Iagaru A (2018) (18)F-florbetaben whole-body PET/MRI for evaluation of systemic amyloid deposition. EJNMMI Res 8(1):66

95. Dorbala S, Vangala D, Semer J, Strader C, Bruyere JR Jr, Di Carli MF, Moore SC, Falk RH (2014) Imaging cardiac amyloidosis: a pilot study using (1)(8)F-florbetapir positron emission tomography. Eur J Nucl Med Mol Imaging 41(9):1652-1662

96. Lee SP, Lee ES, Choi H, Im HJ, Koh Y, Lee MH, Kwon JH, Paeng JC, Kim HK, Cheon GJ, Kim YJ, Kim I, Yoon SS, Seo JW, Sohn DW (2015) 11C-Pittsburgh B PET imaging in cardiac amyloidosis. JACC Cardiovasc Imaging 8(1):50-59

97. Lister-James J, Pontecorvo MJ, Clark C, Joshi AD, Mintun MA, Zhang W, Lim N, Zhuang Z, Golding G, Choi SR, Benedum TE, Kennedy P, Hefti F, Carpenter AP, Kung HF, Skovronsky DM (2011) Florbetapir f-18: a histopathologically validated Betaamyloid positron emission tomography imaging agent. Semin Nucl Med 41(4):300-304

98. O'Keefe GJ, Saunder TH, Ng S, Ackerman U, Tochon-Danguy HJ, Chan JG, Gong S, Dyrks T, Lindemann S, Holl G, Dinkelborg L, Villemagne V, Rowe CC (2009) Radiation dosimetry of beta-amyloid tracers 11C-PiB and 18F-BAY94-9172. J Nucl Med 50(2):309-315

99. Lee S-P, Suh H-Y, Park S, Oh S, Kwak S-G, Kim H-M, Koh Y, Park J-B, Kim H-K, Cho H-J, Kim Y-J, Kim I, Yoon S-S, Seo J-W, Paeng J-C, Sohn D-W (2020) Pittsburgh B compound positron emission tomography in patients with AL cardiac amyloidosis. J Am Coll Cardiol 75(4):380-390

100. Holzmann M, Nicko A, Kuhl U, Noutsias M, Poller W, Hoffmann W, Morguet A, Witzenbichler B, Tschope C, Schultheiss HP, Pauschinger M (2008) Complication rate of right ventricular endomyocardial biopsy via the femoral approach: a retrospective and prospective study analyzing 3048 diagnostic procedures over an 11-year period. Circulation 118(17):1722-1728

101. Yilmaz A, Kindermann I, Kindermann M, Mahfoud F, Ukena C, Athanasiadis A, Hill S, Mahrholdt H, Voehringer M, Schieber M, Klingel K, Kandolf R, Bohm M, Sechtem U (2010) Comparative evaluation of left and right ventricular endomyocardial biopsy: 
differences in complication rate and diagnostic performance. Circulation 122(9):900-909

102. Khan T, Selvakumar D, Trivedi S, Rao K, Harapoz M, Thiagalingam A, Denniss AR, Varikatt W (2017) The value of endomyocardial biopsy in diagnosis and guiding therapy. Pathology 49(7):750-756

103. Frey N, Meder B, Katus HA (2018) Left ventricular biopsy in the diagnosis of myocardial diseases. Circulation 137(19):993-995

104. Kristen AV, Brokbals E, dem Aus SF, Bauer R, Hein S, Aurich M, Riffel J, Behrens HM, Kruger S, Schirmacher P, Katus HA, Rocken C (2016) Cardiac amyloid load: a prognostic and predictive biomarker in patients with light-chain amyloidosis. J Am Coll Cardiol 68(1):13-24

105. Mehta P, Chapel DB, Goyal N, Yu DB, Mor-Avi V, Narang A, Addetia K, Sarswat N, Lang RM, Husain AN, Patel AR (2019) A histopathologic schema to quantify the burden of cardiac amyloidosis: relationship with survival and echocardiographic parameters. Echocardiography 36(2):285-291

106. Fine NM, Arruda-Olson AM, Dispenzieri A, Zeldenrust SR, Gertz MA, Kyle RA, Swiecicki PL, Scott CG, Grogan M (2014) Yield of noncardiac biopsy for the diagnosis of transthyretin cardiac amyloidosis. Am J Cardiol 113(10):1723-1727

107. Cendrowska U, Silva PJ, Ait-Bouziad N, Müller M, Guven ZP, Vieweg S, Chiki A, Radamaker L, Kumar ST, Fändrich M, Tavanti F, Menziani MC, Alexander-Katz A, Stellacci F, Lashuel HA (2020) Unraveling the complexity of amyloid polymorphism using gold nanoparticles and cryo-EM. Proc Natl Acad Sci USA 117(12):6866-6874. https://doi.org/10.1073/pnas.1916176117.

108. Kyle RA, Therneau TM, Rajkumar SV, Larson DR, Plevak MF, Offord JR, Dispenzieri A, Katzmann JA, Melton LJ III (2006) Prevalence of monoclonal gammopathy of undetermined significance. N Engl J Med 354(13):1362-1369

109. Lachmann HJ, Booth DR, Booth SE, Bybee A, Gilbertson JA, Gillmore JD, Pepys MB, Hawkins PN (2002) Misdiagnosis of hereditary amyloidosis as AL (primary) amyloidosis. N Engl J Med 346(23):1786-1791

110. Sattianayagam PT, Hahn AF, Whelan CJ, Gibbs SD, Pinney JH, Stangou AJ, Rowczenio D, Pflugfelder PW, Fox Z, Lachmann HJ, Wechalekar AD, Hawkins PN, Gillmore JD (2012) Cardiac phenotype and clinical outcome of familial amyloid polyneuropathy associated with transthyretin alanine 60 variant. Eur Heart $\mathbf{J}$ 33(9):1120-1127

111. Conceição I, Coelho T, Rapezzi C, Parman Y, Obici L, Galán L, Rousseau A (2019) Assessment of patients with hereditary transthyretin amyloidosis — understanding the impact of management and disease progressio. Amyloid 26(3):103-111

112. Griffiths BE, Hughes P, Dowdle R, Stephens MR (1982) Cardiac amyloidosis with asymmetrical septal hypertrophy and deterioration after nifedipine. Thorax 37(9):711-712

113. Palladini G, Dispenzieri A, Gertz MA, Kumar S, Wechalekar A, Hawkins PN, Schonland S, Hegenbart U, Comenzo R, Kastritis E, Dimopoulos MA, Jaccard A, Klersy C, Merlini G (2012) New criteria for response to treatment in immunoglobulin light chain amyloidosis based on free light chain measurement and cardiac biomarkers: impact on survival outcomes. J Clin Oncol 30(36):4541-4549

114. Schonland SO, Dreger P, de Witte T, Hegenbart U (2012) Current status of hematopoietic cell transplantation in the treatment of systemic amyloid light-chain amyloidosis. Bone Marrow Transplant 47(7):895-905

115. Kastritis E, Wechalekar AD, Dimopoulos MA, Merlini G, Hawkins PN, Perfetti V, Gillmore JD, Palladini G (2010) Bortezomib with or without dexamethasone in primary systemic (light chain) amyloidosis. J Clin Oncol 28(6):1031-1037

116. Venner CP, Lane T, Foard D, Rannigan L, Gibbs SD, Pinney JH, Whelan CJ, Lachmann HJ, Gillmore JD, Hawkins PN,
Wechalekar AD (2012) Cyclophosphamide, bortezomib, and dexamethasone therapy in $\mathrm{AL}$ amyloidosis is associated with high clonal response rates and prolonged progression-free survival. Blood 119(19):4387-4390

117. Varga C, Comenzo RL (2019) High-dose melphalan and stem cell transplantation in systemic AL amyloidosis in the era of novel anti-plasma cell therapy: a comprehensive review. Bone Marrow Transplant 54(4):508-518

118. Maurer MS, Schwartz JH, Gundapaneni B, Elliott PM, Merlini G, Waddington-Cruz M, Kristen AV, Grogan M, Witteles R, Damy T, Drachman BM, Shah SJ, Hanna M, Judge DP, Barsdorf AI, Huber P, Patterson TA, Riley S, Schumacher J, Stewart M, Sultan MB, Rapezzi C (2018) Tafamidis treatment for patients with transthyretin amyloid cardiomyopathy. N Engl J Med 379(11):1007-1016

119. Barroso FA, Judge DP, Ebede B, Li H, Stewart M, Amass L, Sultan MB (2017) Long-term safety and efficacy of tafamidis for the treatment of hereditary transthyretin amyloid polyneuropathy: results up to 6 years. Amyloid 24(3):194-204

120. Adams D, Gonzalez-Duarte A, O'Riordan WD, Yang CC, Ueda M, Kristen AV, Tournev I, Schmidt HH, Coelho T, Berk JL, Lin KP, Vita G, Attarian S, Plante-Bordeneuve V, Mezei MM, Campistol JM, Buades J, Brannagan TH III, Kim BJ, Oh J, Parman Y, Sekijima Y, Hawkins PN, Solomon SD, Polydefkis M, Dyck PJ, Gandhi PJ, Goyal S, Chen J, Strahs AL, Nochur SV, Sweetser MT, Garg PP, Vaishnaw AK, Gollob JA, Suhr OB (2018) Patisiran, an RNAi therapeutic, for hereditary transthyretin amyloidosis. N Engl J Med 379(1):11-21

121. Benson MD, Waddington-Cruz M, Berk JL, Polydefkis M, Dyck PJ, Wang AK, Plante-Bordeneuve V, Barroso FA, Merlini G, Obici L, Scheinberg M, Brannagan TH III, Litchy WJ, Whelan C, Drachman BM, Adams D, Heitner SB, Conceicao I, Schmidt HH, Vita G, Campistol JM, Gamez J, Gorevic PD, Gane E, Shah AM, Solomon SD, Monia BP, Hughes SG, Kwoh TJ, McEvoy BW, Jung SW, Baker BF, Ackermann EJ, Gertz MA, Coelho $\mathrm{T}$ (2018) Inotersen treatment for patients with hereditary transthyretin amyloidosis. N Engl J Med 379(1):22-31

122. Solomon SD, Adams D, Kristen A, Grogan M, Gonzalez-Duarte A, Maurer MS, Merlini G, Damy T, Slama MS, Brannagan TH III, Dispenzieri A, Berk JL, Shah AM, Garg P, Vaishnaw A, Karsten V, Chen J, Gollob J, Vest J, Suhr O (2019) Effects of Patisiran, an RNA interference therapeutic, on cardiac parameters in patients with hereditary transthyretin-mediated amyloidosis. Circulation 139(4):431-443

123. Aus dem Siepen F, Bauer R, Aurich M, Buss SJ, Steen H, Altland K, Katus HA, Kristen AV (2015) Green tea extract as a treatment for patients with wild-type transthyretin amyloidosis: an observational study. Drug Des Devel Ther 9:6319-6325

124. Karlstedt E, Jimenez-Zepeda V, Howlett JG, White JA, Fine NM (2019) Clinical experience with the use of doxycycline and ursodeoxycholic acid for the treatment of transthyretin cardiac amyloidosis. J Card Fail 25(3):147-153

125. Kazi DS, Bellows BK, Baron SJ, Shen C, Cohen DJ, Spertus JA, Yeh RW, Arnold SV, Sperry BW, Maurer MS, Shah SJ (2020) Cost-effectiveness of Tafamidis therapy for transthyretin amyloid cardiomyopathy. Circulation 141(15):1214-1224

126. Real de Asua D, Costa R, Galvan JM, Filigheddu MT, Trujillo D, Cadinanos J (2014) Systemic AA amyloidosis: epidemiology, diagnosis, and management. Clin Epidemiol 6:369-377

127. Okamoto S, Wixner J, Obayashi K, Ando Y, Ericzon BG, Friman S, Uchino M, Suhr OB (2009) Liver transplantation for familial amyloidotic polyneuropathy: impact on Swedish patients' survival. Liver Transpl 15(10):1229-1235

128. Suhr OB, Larsson M, Ericzon BG, Wilczek HE (2016) Survival after transplantation in patients with mutations other than 
Val30Met: extracts from the FAP World Transplant Registry. Transplantation 100(2):373-381

129. Yazaki M, Mitsuhashi S, Tokuda T, Kametani F, Takei YI, Koyama J, Kawamorita A, Kanno H, Ikeda SI (2007) Progressive wild-type transthyretin deposition after liver transplantation preferentially occurs onto myocardium in FAP patients. Am $\mathbf{J}$ Transplant 7(1):235-242

130. Ericzon BG, Wilczek HE, Larsson M, Wijayatunga P, Stangou A, Pena JR, Furtado E, Barroso E, Daniel J, Samuel D, Adam R, Karam V, Poterucha J, Lewis D, Ferraz-Neto BH, Cruz MW, Munar-Ques M, Fabregat J, Ikeda S, Ando Y, Heaton N, Otto G, Suhr O (2015) Liver transplantation for hereditary transthyretin amyloidosis: after 20 years still the best therapeutic alternative? Transplantation 99(9):1847-1854

131. Stangou AJ, Hawkins PN (2004) Liver transplantation in transthyretin-related familial amyloid polyneuropathy. Curr Opin Neurol 17(5):615-620

132. Koike H, Katsuno M (2019) Ultrastructure in transthyretin amyloidosis: from pathophysiology to therapeutic insights. Biomedicines 7(1):11. https://doi.org/10.3390/biomedicines7010011

133. Palladini G, Malamani G, Co F, Pistorio A, Recusani F, Anesi E, Garini P, Merlini G (2001) Holter monitoring in AL amyloidosis: prognostic implications. Pacing Clin Electrophysiol 24(8 Pt 1):1228-1233

134. Varr BC, Zarafshar S, Coakley T, Liedtke M, Lafayette RA, Arai S, Schrier SL, Witteles RM (2014) Implantable cardioverter-defibrillator placement in patients with cardiac amyloidosis. Heart Rhythm 11(1):158-162

135. Sayed RH, Rogers D, Khan F, Wechalekar AD, Lachmann HJ, Fontana M, Mahmood S, Sachchithanantham S, Patel K, Hawkins PN, Whelan CJ, Gillmore JD (2015) A study of implanted cardiac rhythm recorders in advanced cardiac AL amyloidosis. Eur Heart J 36(18):1098-1105

136. Kristen AV, Dengler TJ, Hegenbart U, Schonland SO, Goldschmidt H, Sack FU, Voss F, Becker R, Katus HA, Bauer A (2008) Prophylactic implantation of cardioverter-defibrillator in patients with severe cardiac amyloidosis and high risk for sudden cardiac death. Heart Rhythm 5(2):235-240

137. Lin G, Dispenzieri A, Kyle R, Grogan M, Brady PA (2013) Implantable cardioverter defibrillators in patients with cardiac amyloidosis. J Cardiovasc Electrophysiol 24(7):793-798

138. Patel KS, Hawkins PN, Whelan CJ, Gillmore JD (2014) Lifesaving implantable cardioverter defibrillator therapy in cardiac AL amyloidosis. BMJ Case Rep 2014:bcr2014206600

139. Lin G, Dispenzieri A, Brady PA (2010) Successful termination of a ventricular arrhythmia by implantable cardioverter defibrillator therapy in a patient with cardiac amyloidosis: insight into mechanisms of sudden death. Eur Heart J 31(12): 1538

140. Priori SG, Blomstrom-Lundqvist C, Mazzanti A, Blom N, Borggrefe M, Camm J, Elliott PM, Fitzsimons D, Hatala R, Hindricks G, Kirchhof P, Kjeldsen K, Kuck KH, Hernandez-Madrid A, Nikolaou N, Norekval TM, Spaulding C, Van Veldhuisen DJ (2015) 2015 ESC Guidelines for the management of patients with ventricular arrhythmias and the prevention of sudden cardiac death: The Task Force for the Management of Patients with Ventricular Arrhythmias and the Prevention of Sudden Cardiac Death of the European Society of Cardiology (ESC). Endorsed by: Association for European Paediatric and Congenital Cardiology (AEPC). Eur Heart J 36(41):2793-2867

141. Russo AM, Stainback RF, Bailey SR, Epstein AE, Heidenreich PA, Jessup M, Kapa S, Kremers MS, Lindsay BD, Stevenson LW
(2013) ACCF/HRS/AHA/ASE/HFSA/SCAI/SCCT/SCMR 2013 appropriate use criteria for implantable cardioverter-defibrillators and cardiac resynchronization therapy: a report of the American College of Cardiology Foundation appropriate use criteria task force, Heart Rhythm Society, American Heart Association, American Society of Echocardiography, Heart Failure Society of America, Society for Cardiovascular Angiography and Interventions, Society of Cardiovascular Computed Tomography, and Society for Cardiovascular Magnetic Resonance. J Am Coll Cardiol 61(12):1318-1368

142. Al-Khatib SM, Stevenson WG, Ackerman MJ, Bryant WJ, Callans DJ, Curtis AB, Deal BJ, Dickfeld T, Field ME, Fonarow GC, Gillis AM, Granger CB, Hammill SC, Hlatky MA, Joglar JA, Kay GN, Matlock DD, Myerburg RJ, Page RL (2018) 2017 AHA/ACC/HRS guideline for management of patients with ventricular arrhythmias and the prevention of sudden cardiac death: a report of the American College of Cardiology/American Heart Association Task Force on Clinical Practice Guidelines and the Heart Rhythm Society. J Am Coll Cardiol 72(14):e91-e220

143. Towbin JA, McKenna WJ, Abrams DJ, Ackerman MJ, Calkins H, Darrieux FCC, Daubert JP, de Chillou C, DePasquale EC, Desai MY et al (2019) 2019 HRS expert consensus statement on evaluation, risk stratification, and management of arrhythmogenic cardiomyopathy: executive summary. Heart Rhythm 16(11):e373e407. https://doi.org/10.1016/j.hrthm.2019.09.019

144. Brignole M, Auricchio A, Baron-Esquivias G, Bordachar P, Boriani G, Breithardt OA, Cleland J, Deharo JC, Delgado V, Elliott PM, Gorenek B, Israel CW, Leclercq C, Linde C, Mont L, Padeletti L, Sutton R, Vardas PE, Zamorano JL, Achenbach S, Baumgartner H, Bax JJ, Bueno H, Dean V, Deaton C, Erol C, Fagard R, Ferrari R, Hasdai D, Hoes AW, Kirchhof P, Knuuti J, Kolh P, Lancellotti P, Linhart A, Nihoyannopoulos P, Piepoli MF, Ponikowski P, Sirnes PA, Tamargo JL, Tendera M, Torbicki A, Wijns W, Windecker S, Kirchhof P, Blomstrom-Lundqvist C, Badano LP, Aliyev F, Bansch D, Baumgartner H, Bsata W, Buser P, Charron P, Daubert JC, Dobreanu D, Faerestrand S, Hasdai D, Hoes AW, Le Heuzey JY, Mavrakis H, McDonagh T, Merino JL, Nawar MM, Nielsen JC, Pieske B, Poposka L, Ruschitzka F, Tendera M, Van Gelder IC, Wilson CM (2013) 2013 ESC Guidelines on cardiac pacing and cardiac resynchronization therapy: the Task Force on cardiac pacing and resynchronization therapy of the European Society of Cardiology (ESC). Developed in collaboration with the European Heart Rhythm Association (EHRA). Eur Heart J 34(29):2281-2329

145. Dispenzieri A, Gertz MA, Kyle RA, Lacy MQ, Burritt MF, Therneau TM, McConnell JP, Litzow MR, Gastineau DA, Tefferi A, Inwards DJ, Micallef IN, Ansell SM, Porrata LF, Elliott MA, Hogan WJ, Rajkumar SV, Fonseca R, Greipp PR, Witzig TE, Lust JA, Zeldenrust SR, Snow DS, Hayman SR, McGregor CG, Jaffe AS (2004) Prognostication of survival using cardiac troponins and $\mathrm{N}$-terminal pro-brain natriuretic peptide in patients with primary systemic amyloidosis undergoing peripheral blood stem cell transplantation. Blood 104(6):1881-1887

146. Comenzo RL, Reece D, Palladini G, Seldin D, Sanchorawala V, Landau H, Falk R, Wells K, Solomon A, Wechalekar A, Zonder J, Dispenzieri A, Gertz M, Streicher H, Skinner M, Kyle RA, Merlini G (2012) Consensus guidelines for the conduct and reporting of clinical trials in systemic light-chain amyloidosis. Leukemia 26(11):2317-2325 